\title{
Beiträge zur Physiologie und Anatomie des Cephalopodenauges.
}

\author{
Von \\ C. Hess in Würzburg. \\ (Hierzu Tafel V-VIII.) \\ I.
}

Von mehreren Forschern, die sich mit dem Studium des Cephalopodenauges beschäftigt haben, wurde eine rote Färbung der Netzhautstäbchen erwähnt, zuerst 1839 von $\mathrm{A}$. Krohn ${ }^{1}$ ), später von Hensen ${ }^{2}$ ), Max Schultze ${ }^{3}$ ) und Anderen. Max Schultze machte über die Netzhautfarbe folgende Angaben: „Die Farbe, in welcher sich an solchen (sc. in Schälchen mit Jodserum liegenden) Präparaten die Retina zeigt, ist nach Arten und Individuen verschieden, bald dunkel schwarzbraun, bald blass rosenrot, je nach der bereits von früheren Beobachtern beschriebenen verschiedenen Pigmentierung der Stäbchenschicht. Die rosenrote Farbe beruht auf einer diffusen Färbung der ganzen Dicke der Stäbchenschicht, ist aber nur an frischen Exemplaren sichtbar, wo ihrer schon Krobn Erwähnung tut. Mit dem Mikroskop ist sie nur an dickeren Schichten abgelöster Stäbchen erkennbar. Dieselbe kann für die Betrachtung Init blossem Auge mehr oder weniger vollständig verdeckt werden durch die braunschwarzen, körnigen Pigmentierungen, welche sich bei manchen Arten innerhalb der Stäbchenschicht vorfinden. . . . Fast ganz pigmentfrei und von der schönsten rosenroten Farbe sah ich die Stäbchenschicht im Auge eines grossen Exemplars von Loligo sagittata."

1) A. Trohn, Nachtr. Bemerkungen über den Bau des Cephalopodenauges. Verhandl. d. Leop.-Carol. Akad. Bd. 19 S. 45.1842.

2) Hensen, Über das Auge einiger Cephalopoden. Zeitschr. f. wissensch. Zool. Bd. 15. 1865. - Derselbe, Über den Sehpurpur bei Mollusken. Zool. Anzeiger Bd. 1 Nr. 2 S. 30. 1878.

3) M. Schultze, Die Stäbchen in der Retina der Cephalopoden und Heteropoden. Arch. f. mikrosk. Anat. Bd. 5. 1869.

Đ. Pflüger, Archiv für Physiologie. Bd. 109. 
Nachdem der Sehpurpur in Wirbeltierauge dureh Boll und Kühne (1876) entdeckt und studiert worden war, wurde Krukenberg von Letzterem zu einer Untersuchung der Cephalopodenuetzhaut auf das Vorkommen von Sehpurpur angeregt. Er berichtete in einem Briefe an $K u ̈ h n e^{x}$ ) ubber Versuche, bei welchen lebende Exemplare von Loligo auf dunklem Grunde einseitig belichtet wurden: "Die Farbe der Stäbchen beider Augen liess in den angestellten Versucbsreihen absolut keinen Unterschied erkennen, und es darf somit behauptet werden, dass der Stäbchenpurpur der Cephalopoden ebensowenig lichtempfindlich ist, wie nach Ihren Untersuchungen derjenige von Astacus. Auch an Sepia wurde dieser Versuch ausgeführt, und zwar mit dem nämlichen Erfolge. .... Der Stäbchenpurpur ist nicht nur sehr resistent dem Licht gegenüber, wovon ich mich ausser am lebenden Tiere noch an der herausgenommenen Retina, welche mehrere Stunden in einer Kochsalzlösung dem Sonnenlicht exponiert und durch dasselbe nicht bemerkbar verändert wurde, überzengt habe, sondern er erträgt auch eine ziemlich hohe Temperatur."

Weitere Untersuchungen über die Färbung der Cephalopodennetzhaut sind mir nicht bekannt geworden. Noch im Jahre 1900 machte R. Greeff ${ }^{2}$ ) die Angabe, dass bei allen Wirbellosen der Sehpurpur fehle; "die zahlreichen, zum Teil auch roten Pigmente, welche bei den Wirbellosen vorkommen, sind ganz anderer Natur und sind im Licht nur sehr langsam vergänglich." Speciell für die rote Farbe der Cephalopodennetzbaut wurde der Meinung Ausdruck gegeben, dass sie nicht durch Sehpurpur bedingt sei.

Demgegenüber konnte $\mathrm{ich}^{3}$ ) den Nachweis erbringen, dass sich in den Netzhäuten verschiedener Cephalopodenarten ein sehr lichtempfindlicher, anscheinend dem Sehpurpur der Wirbeltiere nahestehender roter Farbstoff vorfindet.

Im folgenden berichte ich kurz über weitere Untersuchungen, die ich inzwischen an Cephalopodenaugen in Neapel anstellen konnte. Herrn Geheimrat Dohrn danke ich auch hier für das Entgegenkommen, das ich bei ihm und an der zoologischen Station gefunden habe; Herrn Cav. Lo Bianco bin ich für seine liebenswürdige Unterstützung und Versorgung mit Material zu Danke verpflichtet.

1) Untersuchungen aus dem physiol. Institut Heidelberg 1882.

2) Handb. d. ges. Augenheilkunde von $S a ̈ m$ is ch Bd. 1 (1) Kap. V S. 111.

3) Zentralbl. f. Physiol. 1902 H. 4. 
Die hauptsächlichsten Schwierigkeiten bei Untersuchung des Sehpurpurs der Cephalopoden bietet die Anwesenheit von Pigment in der Stäbchenschicht selbst; hierauf ist es wohl auch wesentlich zurückzuführen, dass die merkwürdigen Eigenschaften, vor allem die Lichtempfindlichkeit dieses Farbstoffes so lange ganz unbekannt geblieben sind.

Beim Wirbeltier ist es sehr leicht, insbesondere an Dunkelaugen die Netzbaut so gut wie vollständig frei von Pigment zu erhalten; bei den Cephalopodenaugen ist dies unmöglich, einmal, weil bei mebreren Arten selbst nach längerer Verdunklung Pigment noch iı grosser Menge nahe der vitralen Oberfläche der Netzbaut sich findet (vergl. Abschnitt 3), dann, weil bei allen hier in Betracht kommenden Arten das Pigment an der Basis der Stäbchen mit diesen innig verbunden, daher eine mechanische Trennung der letzteren vom Pigmente, wie im Wirbeltierauge, nicht möglich ist.

Man sieht daher auch bei günstigsten Verhältnissen hinsichtlich der Pigmentstellung die Netzhaut stets auf tiefschwarzer Unterlage, wodurch die Beobachtung einer Färbung der im Leben ziemlich durchsichtigen Netzhaut unter den gewöhnlichen Bedingungen fast unmöglich ist.

Bei meinen Untersuchungen schlug ich zur Ermittelung der fraglichen Färbungen die folgenden Wege ein: Finmal wurde die frische Netzhaut derart in Falten gelegt, dass Stellen ohne vitrales Pigment (siehe unten) die Kuppe von Falten bildeten, auf welche ich schräg von der Seite blickte. Ferner wurden die Schnittfächen von Querschnitten durch die beí vielen Arten besonders dicken und oft pigmentfreien mittleren Netzhautteile (siehe unten) auf ihre Färbung unter den verschiedensten Verhältnissen geprüft. Eine dritte, für viele Zwecke geeignete Untersuchungsmethode bestand darin, die Netzhaut durch passende, den Farbstoff nicht angreifende Reagenzien leicht $\mathrm{zu}$ trüben; der dunkle Pigmentgrund stört dann weniger oder gar nicht mehr, während die Färbung der Stäbchenschicht, solange die Trübung der letzteren zart genug bleibt, deutlich bervortritt. Viertens endlich habe ich vielfach Zupfpräparate unter verschiedenen Versuchsbedingungen und Belichtungsverhältnissen mikroskopisch untersucht.

Mit diesen Methoden konnte ich Sehpurpur in den Netzhäuten folgender Arten nachweisen: Sepia, Sepiola, Eledone, Octopus, Loligo, Rossia, Scaeurgus, Todaropsis; aus der ziemlich grossen Zahl meiner 
einschlägigen Beobachtungen gebe ich hier nur die wichtigsten etwas ausführlicher wieder.

Als verhältnismässig günstiges Untersuchungsobjekt erwies sich mir Loligo (L. vulgaris und L. marmorae), die mir in grossen Mengen zur Verfügung stand und deren Pigment, wenigstens in den mittleren Netzhautteilen, bei schwachem Lichte oder nach kurzer Verdunklung im allgemeinen genügend scleral liegt, um eine Beobachtung der Stäbchenfärbung ohne zu grosse Schwierigkeiten zu gestatten.

Eröffnet man bei möglichst schwachem Tageslichte ${ }^{1}$ ) das frische Auge einer längere Zeit im Dunkeln gehaltenen Loligo, so erscheint, insbesondere wenn die Blickrichtung unter spitzem Winkel die Ebene der Netzhaut trifft, letztere meist ungefähr in ihren oberen zwei Dritteln ${ }^{2}$ ) schön dunkelrot bis purpurfarbig, in ihrem unteren Drittel meist noch wesentlich dunkler als im oberen, oft fast schwarz; doch konnte ich bei einzelnen, ca. fünf Stunden dunkel gehaltenen Exemplaren deutliche Purpurfärbung auch an diesem unteren Teile nachweisen. Der Unterschied in der Helligkeit zwischen den oberen und den unteren Netzhautteilen war im allgemeinen um so geringer, je frischer das Auge (je durchsichtiger also wohl die Netzhaut) war. Wird nun ein Stäck dieser Netzhaut an die Sonne gebracht, so ist oft schon in weniger als einer Minute die Purpurfarbe in ein ungesättigtes bräunliches Gelb ohne Spur von Rot übergegangen; weiterhin wird die Farbe allmählich mehr strohgelb.

Bei derartigen Versuchen wurde stets ein Kontrollstück der Netzhaut im Dunkeln gehalten und wiederholt bei möglichst schwachem diffusen Lichte die Farbe beider Stücke direkt verglichen. Bei Anlegen von Schnitten muss auf die Möglichkeit einer verschiedenen Färbung der verschiedenen Netzhautpartien entsprechend Rücksicht genommen werden. Da im allgemeinen (s. u.) die Färbung der Netzhäute an den verschiedenen Stellen eines wagerechten Schnittes die gleiche ist, dagegen an versehiedenen Stellen eines senkrechten Schnittes beträchtlich variieren kann, so sollen die Trennungsschnitte $\mathrm{zu}$ den fraglichen vergleichenden Versuchen senkrecht angelegt werden; in der Regel wurden die Augen durch einen

1) Diese Versuche wurden in einem Dunkelzimmer angestellt, das eine innerhalb weiter Grenzen variable Fensteröffnung besass, die noch mit Mattgläsern und nach Bedürfnis mit farbigen Gläsern verdeckt werden konnte.

2) Genaueres über die hier in Betracht kommenden Pigmentverhältnisse siehe im dritten Abschnitte, 
solchen Schnitt in zwei seitliche Hälften geteilt, die bei gleichen Beleuchtungsbedingungen übereinstimmende Färbung zeigten.

Die nach Schwinden der Purpurfärbung sichtbare gelbe Farbe pflegt im allgemeinen wesentlich lichtbeständiger zu sein als das Rot: bei einem Loligo-Auge fand ich nach $3 / 4$ Stunden dauernder Besonnung die Farbe der Netzhaut noch deutlich gelblich.

Bei diffusem Lichte erfolgt der Übergang aus Rot in Gelb entsprechend langsamer. Zerzupft man bei möglicbst schwachem Lichte rasch ein Stück Dunkelnetzhaut (z. B. einer Loligo), so erscheinen unter dem Mikroskop die Stäbchen zunächst schön purpurrot, nach einigen Minuten diffuser Belichtung (ohne direkte Sonne) blassgelb, in dünneren Schichten nahezu farblos. Diese Gelbfärbung war auch nach zwei bis drei Stunden dauernder Einwirkung diffusen Lichtes fast unverändert nachweisbar. Kontrollversuche wurden in der Weise angestellt, dass ein Teil des Objektträgers beschattet, der andere belichtet wurde; die verschiedene Färbung der beiden Teile unter dem Mikroskop war schon nach sehr kurzer Zeit leicht wahrzunehmen.

Wurde eine Dunkelnetzhaut durchschnitten, so ersehien die Schnittfläche als leuchtend purpurrote oder gelblichrote Linie, deren Färbung am Lichte rasch in Gelb überging.

Die Purpurfärbung der Stäbchen konnte ich ebensowohl an jenen Netzhautpartien nachweisen, die keine Lichtstellung des Pigmentes zeigten, als an jenen mit ausgesprochener innerer Pigmentzone.

Während bei Loligo die direkte Beobachtung der Netzhautfärbung unter den genannten Versuchsbedingungen in der Regel ziemlich leicht ist, wird bei versehiedenen anderen Cephalopodenarten die Erkennung des Sehpurpurs erst auf Umwegen möglich.

Die Netzhäute von Sepia officinalis erschienen, wenn das Auge ganz frisch eröffnet wurde, oft tiefdunkel oder schwarz, so dass eine Färbung gar nicht oder nicht mit genügender Sicherheit sich feststellen liess; auch nach Besonnung eines Netzhautstïckes konnte zunächst zwischen diesem und dem dunkel gehaltenen Auge kein deutlicher Unterschied festgestellt werden, sie ersehienen beide schwarz. Wurden aber jetzt beide Stücke in $4 \%$ iges Formolseewasser gebracht, so trat bald eine leichte Trübung der Netzhäute auf, und es erschien nun die Dunkelnetzhaut schön rot, zuweilen mit einem Stiche ins Violette, die Hellnetzhaut dagegen graugelb; wurde dann 
ein Stück der Dunkelnetzhaut im Formol an die Sonne gebracht, so ging die Purpurfarbe bald in Graugelb über. Auf Querschnitten durch die Netzhaut zeigten besonders die mittleren, dicken Teile am Formolpräparat eine schön rote Farbe, die nach 10-15 Minuten dauernder Einwirkung diffusen Lichtes in Gelb übergegangen war.

Die Purpurfärbung hält sich im Formol geraume Zeit, oft mehrere Tage lang, ziemlich unverändert, wenn das Auge vor Belichtung geschützt bleibt. Eine Hell- und eine Dunkelnetzhant, die in Formol 24 Stunden im Dunkeln gehalten worden waren, zeigten den charakteristischen Farbunterschied noch fast ebenso schön wie kurz nach Einlegen in Formol; eine andere Dunkelnetzhaut (von Rossia) war nach zweitägiger Formolwirkung noch graurot, das Rot ging an der Sonne in einer bis zwei Minuten in Graugelb über. (Doch darf man, wie es scheint, daraus nicht schliessen, dass das Formol allgemein ganz ohne Einfluss auf die Stäbchenfarbe sei: Eine gelbe Hellnetzhaut und eine rote Dunkelnetzhaut wurden in Formol im Dunkeln gelassen; nach einem Tage fand ich die Hellnetzhaut farblos grau, die Dunkelnetzhaut nur noch schwach rötlichgelb.)

Ein Stück Dunkelnetzhaut, das 24 Stunden in Formol gelegen hatte und schön rot erschien, wurde an der Sonne erst nach ca. fünf bis zehn Minuten deutlich graugelb, während das dunkel gehaltene Vergleichsstück noch leuchtend rot erschien. Nach diesen Versuchen hat es den Anschein, als ob hier das Formol die Lichtempfindlichkeit des Sehpurpurs abgestumpft hätte. (Für den Wirbeltiersehpurpur hat ein Gleiches bei Anwendung von $4-10 \%$ igen Formalinlösungen Abelsdorff angegeben.)

Durch freundliche Vermittlung der zoologischen Station in Helgoland wurden mir die Augen eines im Dunkeln gefangenen sehr grossen Exemplars von Loligo Forbesii zugeschickt, die sofort in $5 \%$ iges Formol gebracht worden waren; die Hälfte eines Auges wurde längere Zeit an einem trüben Tage dem diffusen Lichte ausgesetzt, die andere dunkel gehalten; auch hier zeigte sich noch ein deutlicher Farbenunterschied; die Hellnetzhaut war gleichmässig hellgrau, die Dunkelnetzhaut noch deutlich rötlich; insbesondere war der Unterschied auch auf den Netzhautquerschnitten schön wahrnehmbar. Analoge Beobachtungen konnte ich an Augen von Octopus macropus machen, die ich der freundlichen Vermittlung von Herrn Professor von David off in Villafranca verdanke: die in $4 \%$ igem Formolwasser fixierten Netzhäute erschienen unmittelbar nach Er- 
öffnung der Augen rotgrau; am Lichte ging der rote Farbenton bald verloren.

Bei nicht mehr ganz frischen Augen, z. B. solchen einer vor mehreren Stunden gestorbenen Sepia, kann die Netzhaut auch ohne Einwirkung von Reagenzien schön rot erscheinen, anscheinend infolge leichter kadaveröser Trübung ihres Gewebes. Dieses Rot geht unter dem Einflusse des Lichtes verhältnismässig rasch in Gelb über; an der Sonne ist meist der Farbuntersehied schon nach einer bis zwei Minuten sehr gross. Eine Netzhaut wurde mittels Lupe etwas stärker belichtet (selbstverständlich nicht etwa so, dass das Sonnenbild auf die Netzhautebene zu liegen kam); der belichtete Teil erschien schon nach wenigen Sekunden graugelb. Das Gelb ist auch hier wieder viel lichtbeständiger, die Netzhaut erscheint selbst nach zweistündiger Besonnung noch gelblich, nicht ganz farblos.

Die Farbenänderungen, die ich bei den anderen oben genannten Cephalopodenarten unter dem Einflusse des Lichtes beobachtete, waren im wesentlichen ähnliche oder die gleichen wie bei Loligo und Sepia, so dass ich auf eine ausführliche Beschreibung des Sehpurpurs einer jeden Art verzichten kann.

Ich stelle hier die für uns wichtigsten Tatsachen über das Verhalten des Wirbeltiersehpurpurs nach Kühne (Hermann's Handb. d. Physiol. Bd. 3 Heft 1 S. 288 ff.) zusammen: „Ans Tageslicht gebracht schlägt die Netzhautfarbe entweder allmählich in röteren Purpur, reines Rot, Orangegelb und Chamois um, ehe sie vollkommen farblos wird, oder sie geht mit einem Schlage durch blasses Lila zur Farblosigkeit über. Je frischer die Netzhaut und je lichtempfindlicher infolge davon, aus noch zu erörternden Gründen, der Purpur ist, desto mehr ist die letztere Erscheinung ausgeprägt ... Froschnetzhäute, obwohl in direktem Sonnenlichte anscheinend momentan bleichend, lassen fast immer zuvor ein gelbes oder chamoisfarbenes Stadium deutlich aufkommen. Schneller als die Netzhant wird die Purpurlösung gebleicht, und in dieser ist Chamoisfarbe oft kaum kenntlich, während die gelbe nur an direktem Sonnenlichte zu übersehen ist." "Ans Tageslicht gebracht zeigt die Lösung die Farbe nur einige Augenblicke, indem sie schleunigst rot, darauf gelb, zuletzt farblos wird wie Wasser." ${ }^{1}$ ) "Augenscheinlich ist die Wirkung

1) Dagegen wird von Köttgen und Abelsdorff das Vorkommen von Sehgelb bei den Wirbeltieren entschieden in Abrede gestellt; der Sehpurpur erbleiche, ohne in eine anders gefärbte Substanz überzugehen. (Zeitschr. f. Psych. u. Physiol. d. Sinnesorgane Bd. 12 S. 161.) 
abhängig von der Intensität des Lichtes, langsam verlaufend bei schwacher Belenchtung; bei zur Frkennung der Zwischenfarben gerade ausreichendem Lichte nimmt sie 15-20 Minuten in Anspruch."

Will man die Lichtempfindlichkeit des Cephalopodensehpurpurs mit jener des Wirbeltierpurpurs vergleichen, so sind die folgenden Umstände zu berücksichtigen.

Nach $\mathrm{K} u ̈ h n e$ hat die „anscheinende Indolenz des Sehpurpurs im Leben" ihre Ursache in der Wiedererzengung des Purpurs vom retinalen Epithel aus. "Während die Froschnetzhaut isoliert im direkten Sonnenlicht in unmessbar kurzer Zeit ausbleicht, hedurf sie im Auge des lebenden Frosches dazu mindestens zehn Minuten und noch etwa drei Minuten, wenn Kornea und Linse entfernt sind und die Sonne durch das grosse Loch darauf scheint, das sich an Stelle der engen Pupille durch einen Sperrhalter in der Iris herstellen lässt. Ein im diffusen Tageslicht lang geöffnet gelegener Bulbus liefert häufig noch recht intensiv gefärbte Netzhautpräparate".

Bei den Cephalopodenaugen ist die regenerierende Zelllage fest und untrennbar mit den Stäbchen verbunden; daher sind die Regenerationsbedingungen hier, soweit die Beziehungen zum Pigmente in Betracht kommen, verhältnismässig sehr günstig. Ferner ist zu berücksichtigen, dass die purpurhaltige Stäbchenschicht bei den Cephalopoden durcbschnittlich über $0,2 \mathrm{~mm}$, also ungefähr $3-4$ mal so dick ist, wie im Wirbeltierauge, wo die Stäbchen eine mittlere Länge von 0,05 bis $0,06 \mathrm{~mm}$ besitzen.

Die Vermutung, dass die innige Verbindung der Pigmentlage mit den Stäbchen vielleicht in ähnlicher Weise, wie es $\mathrm{Kühne} \mathrm{bei} \mathrm{den} \mathrm{hier}$ zitierten Beobachtungen für das Wirbeltierange beschreibt, für die Sehpurpurneubildung bei den Cephalopoden wirksam ist, findet wohl eine Stütze in der von mir gemachten Beobachtung (s. u.), dass der Sehpurpur solcher Augen, die eine Zeit in Alaun oder Kochsalzlösungen gelegen hatten und dadurch wohl der Regenerationsfähigkeit beraubt waren, sehr rasch ausbleichte. Weiter ist hier noch folgende Angabe Kühne's von Interesse: „Manche Netzhäute werden am Liehte auffallend spät farblos, indem der Purpur zwar wie gewöhnlich schnell umschlägt, das rote und orange Stadium aber sehr verlängert wird und das letzte Gelb oft stundenlang zerstreutem, gutem Tageslichte standhält. Tritt die Erscheinung an frischen Netzhäuten auf, so deutet sie auf eine, namentlich bei Fröschen vorkommende Abnormität, die sich meist schon in der ungewöhnlich wenig purpurnen 
Farbe der Dunkelretina zu erkennen gibt. Beim Aal und bei der Eule wurde Ähnliches gesehen."

Bei mehreren Cephalopodenarten zeigte sich eine rote Färbung der Netzhaut nicht nur, wenn das Tier im Dunkeln getötet war, sondern auch dann, wenn es sich vorher in diffusem Lichte befunden hatte. Zur Beurteilung dieser Verhältnisse hat die Kenntnis des Pupillenspieles bei den Cephalopoden ein gewisses Interesse.

Beer gibt an, dass die litoralen Cephalopoden in einem bellen Raume die Pupillen geschlossen haben; selbst in einem mässig hellen Raume erinnere er sich, Octopus macropus tagsüber nie anders gesehen zu haben, als mit festverschlossener Pupille und auch Sepia und Eledone nie mit weit offener, meist mit schmalspaltiger. "Die Pupillen der pelagischen Arten, wie Illex, Todarodes, Loligo usw. schliessen sich auch im hellen licht nicht." Bezüglich der litoralen Formen habe ich das Gleiche beobachtet wie Beer, dagegen habe ich wiederholt gesehen, das frische und lebhafte Exemplare von Loligo nicht nur an der Sonne, sondern auch bei diffusem Tageslichte ihre Iris in der sog]eich $\mathrm{zu}$ schildernden Weise derart zusammenzogen, dass von der Pupille so gut wie nichts zu sehen war. Erst wenn die Tiere anfingen, matt zu werden, fand ich auch bei direkter Besonnung die Pupillen offen. Bei Sepia beobachtete ich mehrfach, dass die Tiere in dem der Sonne ausgesetzten Bassin sich so drehten, dass möglichst wenig Licht in die Augen fiel.

Ein Scaeurgus unicirrus heftete sich unter gleichen Verbältnissen so an den Rand des Bassins fest, dass seine durch eine breite Haut untereinander verbundenen Arme einen Schirm zu bilden schienen, in dessen Schatten das Tier sass. Doch habe ich den Versuch nicht genügend häufig wiederholen können, um hier die Möglichkeit eines Zufalles sicher auszuschliessen.

Jedenfalls geht aus dem Mitgeteilten hervor, dass viele Cephalopoden durch Pupillenverengerung ihre Augen viel vollständiger vor Lichteinfall zu schützen vermögen als ein grosser Teil der Wirbeltiere, und dass schon verbältnismässig nicht sehr hohe Lichtstärken genügen, um einen derartig vollständigen Lichtschutz eintreten zu lassen.

Weiter bestehen bei manchen Cepbalopodenarten zweckmässige Einrichtungen zum Schutze gegen das vorwiegend von oben einfallende Licht. Bei Loligo z. B. wird bei Belichtung der obere Teil der Iris wie ein Vorhang so weit über die Linse heruntergezogen, dass ein beträchtlich unterhalb des vorderen Linsenscheitels gelegener 
wagerechter, leicht nach unten konvexer Schlitz entsteht; ist dieser nur wenig geöffnet, so kann wohl von unten und von der Seite her, nicht aber von oben Licht ins Auge fallen. Die Färbung der Iris kann durch Änderung des Zustandes der in ihr enthaltenen Chromatophoren beträchtlich variieren (der dabei in Betracht kommende Innervationsmechanismus ist kürzlich in einer interessanten Untersuchung von R. Magnus aufgedeckt worden). -

Um weitere Eigenschaften des Cephalopodensehpurpurs zu ermitteln und um festzustellen, inwieweit eine Übereinstimmung bezw. Verschiedenheit rom Wirbeltiersehpurpur bestehe, habe ich folgende Versuche angestellt:

Postmortale Veränderungen sind offenbar nicht von beträchtlichem schädigenden Einflusse auf den Cephalopodenpurpur: Netzhäute von Loligo, die ich nach der Enukleation zwei Tage im Dunkeln gehalten hatte, bildeten eine feuchte, breiartige Masse von grauroter Farbe, die am diffusen Lichte eines sehr trüben Regentages in wenigen Minuten in Graugelb überging. Auch Netzhäute von Sepia, die vier Tage im Dunkeln feucht gelegen hatten, zeigten noch schön rote Farbe, während die Hellnetzhäute graugelb erschienen. (Für Wirbeltiere fand Kühne, dass faulende Netzhäute bei sorgfältigem Lichtschutze auch nach Monaten nicht an Färbung verlieren.)

Für den Einfluss der Temperatur auf den Wirbeltiersehpurpur gibt $K u ̈ h n e$ an, dass (feuchte) Froschnetzhäute bei $76^{\circ} \mathrm{C}$. augenblicklich, bei $75^{\circ}$ nach einer Minute, bei $70^{\circ}$ in acht Minuten, bei $60^{\circ}$ in einer Stunde entfärbt werden.... Zusatz von Natriumkarbonat oder $\mathrm{NH}_{3}$ setzt die Entfäròungstemperatur für die Netzhaut bedentend, auf etwa $47^{\circ}$ herab. Purpurlösungen aus Kaninchennetzhäuten werden schon bei $62^{\circ}$ in zweieinhalb Minuten entfärbt, bei $58^{\circ}$ in vier Minuten chamois. „Es gibt da also auf chemische Unterschiede des Purpurs in der Tierreihe, - wofür sich noch viele andere Gründe finden -, deutende Tatsachen."

Ich erwärmte Stücke einer schön roten Dunkelnetzhaut von Loligo langsam im Wasserbade bei sehr schwachem Lichte; bei $55^{\circ}$ C., in andern Versuchen bei ca. $50-52^{\circ}$, wurden sie graurot, trübe; ein Stück hiervon an die Sonne gebracht wurde rasch graugelb. Die bei $55^{\circ}$ graurot gewordenen Stücke wurden bei $58^{\circ}$ bald gelb und stets bei $60^{\circ}$ farblos grau.

Eine Reihe weiterer Versuche wurde veranlasst durch die von 
Kühne mitgeteilte Tatsache, dass der Vertebratensehpurpur von Al a u nicht alteriert wird.

Das Auge einer Sepia, die während der Nacht gestorben war, wurde im Dunkeln eröffnet und in (4\% Kali-)Alaun gebracht; dabei wurde sie bald graurot und erschien mit einem zarten weissen Schleier bedeckt; die eine Hälfte dieser Netzhaut ist an der Sonne nach einer bis zwei Minuten weiss mit einem Stich ins Gelbe, während die Dunkelnetzhaut schön rot bleibt. Nach Entfernung der (sich nicht in toto ablösenden) Membrana limitans ist die Netzhaut schön graurot zu sehen, sie zeigt an der Sonne schon nach zehn Sekunden deutliche Gelbfärbung. Nach zehn Minuten ist sie fast farblos.

Die Netzhaut eines Octopus, der drei Tage dunkel gehalten war, erscheint frisch gleichmässig tiefdunkelbraun; ein Teil davon wird in Alaun ans Helle (nicht direkte Sonne) gebracht, ein anderer in Alaun dunkel gehalten. Zunächst ist zwischen beiden Stücken kaum ein Unterschied zu sehen. Nach einer Viertelstunde ist die Dunkelhälfte rot, die Hellhälfte viel mehr gelb.

Der hintere Abschnitt des Auges einer durch 87 Stunden dunkel gehaltenen Sepia wird in Alaun gelegt; die Netzhaut erscheint zunächst tiefschwarz, auf Falten schön purpurrot. Sie trübt sich in den nächsten Minuten, während deren sie bei stark herabgesetzter Beleuchtung beobachtet wird, wobei die Purpurfarbe sehr deutlich hervortritt. Bei dem diffusen Lichte des bedeckten Himmels geht diese sehr bald durch Gelbrot in Gelb über; nach ungefähr einer Stunde ist die Netzhaut blass gelblich grau, während die Dunkelnetzhaut noch schön graurot ersebeint. Unter dem Mikroskop zeigt sich an Zupfpräparaten die Stäbchenschicht gleichmässig schön rot; auch hier ist der Übergang in Strohgelb unter dem Einfusse des Lichtes schön zu verfolgen. Nach mehreren Stunden ist die Hellnetzbaut blass strohgelb, auch die Dunkelnetzhaut etwas blasser geworden, aber noch graurot.

Von einer schon mehrere Stunden toten Loligo wird die bei schwachem Lichte präparierte gleichmässig purpurrote Netzhaut zur einen Hälfte besonnt und erscheint schon nach 20 Sekunden deutlich gelb neben der mehr bläulichroten Dunkelnetzhaut. In Alaun zeigen beide bald einen grauen Schimmer, doch ist der Farbunterschied noch sehr gross. Bei weiterer Besonnung wird die Hellnetzbaut in Alaun nach ca. einer Viertelstunde blassstrohgelb, nach ca. $1-1^{1 / 2}$ 
Stunden farblos, hellgrau. Die Dunkelnetzhaut wird bei Besonnung nach einiger Zeit graugelb.

Das Auge einer schon einige Stunden toten Loligo wird in Seewasser zur einen Hälfte durch vier Stunden diffus belichtet, zur anderen dunkel gehalten, dann werden beide in Alaun gebracht; es zeigt sich bald ein grauer Schimmer auf beiden Netzhäuten, die dunkel gehaltene erscheint schön rot, die diffus belichtet gewesene hellgelb.

Die Hoffnung, dass es gelingen würde, in ähnlicher Weise, wie bei Wirbeltieren den Sehpurpur mit Hilfe des Alauns zu erhalten, erfüllte sich nicht; bei längerer Alaunwirkung änderte der Cephalopoden-Sehpurpur auch bei Lichtabschluss seine Farbe; in dem zuletzt angeführten Falle war $2^{1 / 2}$ Stunden nach Beginn der Alaunwirkung auch an dem dunkel gehaltenen Stücke das Rot in Gelb übergegangen; das andere (nach der Belichtung gleichfalls ins Dunkle gebrachte) Stück erschien nur ein wenig blasser gelb als das erste.

Die Farbe des Wirbeltiersehpurpurs bleibt ausser in Alaun u. a. auch in Chlornatrium jeder Konzentration, in Ammoniak und in kohlensaurem Alkali unverändert. Für Chlornatri um trifft dies auch beim Cephalopoden-Sehpurpur zu: Die schön purpurrot erscheinende Netzhaut einer Loligo wird in konzentrierte Chlornatriumlösung gelegt und die eine Hälfte davon ans Helle gebracht; nach wenigen Minuten ist die Hellnetzhaut braungelb, nach ca. einer Stunde Sonne grau mit einem Stich ins Braune; die andere, dunkel gehaltene Hälfte erscheint noch nach zwei Tagen sebön rot und wird dann an der Sonne in einer bis zwei Minuten gelb. Bei einem zweiten, ähnlichen Versuche (Loligo) wurde die in konzentriertem Chlornatrium liegende rote Dunkelnetzhaut an der Sonne in wenigen Sekunden gelblich rot, nach ca. einer Minute war sie braungrau, nach drei Stunden farblos grau, während das im Dunkeln gehaltene Stück der in Chlornatrium liegenden Netzhaut noch nach zwei Tagen leuchtend rot erschien.

In einer starken (nicht konzentrierten) Kochsalzlösung erschien ein länger belichtetes Netzhautstück grau, ein dunkel gehaltenes rot; der Farbunterschied war an diesen Stücken noch schön zu sehen, nachdem beide acht Tage lang im Dunkeln in der Kochsalzlösung gelegen hatten.

Träufelt man auf ein Stück schön rot erscheinender Loligo netzhaut einen Tropfen stark verdünnter Salzsäure, so wird die Stelle fast momentan weisslich gelb; ebenso kann man das Gelb- 
werden bei Salzsäurezusatz an Zupfpräparaten unter dem Mikroskop verfolgen. Bei Zusatz von $70 \%$ igem Alkohol zu einer frischen oder mit Formol vorbehandelten, noch rot erscheinenden Netzhaut wird diese sofort graugelb.

Auch bei Aufträufeln von kohlensaurem Natron oder von Ammoniak wird die rote Stäbchenfarbe fast momentan zerstört. Krukenberg's Angabe, der rote Farbstoff werde von $\mathrm{NH}_{3}$ unverändert gelöst, ist demnach unrichtig; der Irrtum wurde offenbar dadurch veranlasst, dass, wie ich fand, Ammoniak, wie überhaupt viele alkalische Flüssigkeiten, auch in starker Verdünnung das Pig ment der Cephalopodennetzhaut rasch unter Bildung einer schön roten, aber lichtbeständigen Flüssigkeit auflöst. (Ebenso wird das Pigment auch durch Säuren gelöst, wie Grenacher ${ }^{1}$ ) fand und ich auch für sehr verdünnte Säuren bestätigen kann.) Auch von Netzhäuten, die fast zwei Jahre lang in Alkohol gelegen hatten, erhielt ich bei Zusatz von stark verdünntem Alkali in wenigen Minuten schön rote Pigmentlösungen; wurde von diesen ein Teil im Dunkeln, ein anderer im Hellen gehalten, so war nach zwei bis drei Tagen diffuser Belichtung noch keine Spur von Farbunterschied zu sehen; erst bei langem Stehen im Hellen blasste die Lösung langsam etwas ab.

An den mit Ammoniak oder kohlensaurem Natron behandeiten Zupfpräparaten sieht man auch bei verdünnten (z. B. 1\% igen) Lösungen schon nach wenigen Sekunden in der Umgebung des Pigmentes eine braunrote Flüssigkeit auftreten, die durch Auflösung des Pigmentes entsteht. Kleine Stückchen einer Cephalopodennetzhaut können in $1 \%$ iger Lösung von Ammoniak oder kohlensaurem Natron schon in wenigen Stunden bei gut erhaltener Strulstur vollständig entpigmentiert sein. Das mobile und das stabile Pigment (s. u.) lösen sich anscheinend in gleicher Weise.

Es wäre auf dem hier angedeuteten Wege vielleicht möglich, bei systematischen Untersuchungen ein geejgnetes Lösungsmittel für das Pigment zu finden, das etwa in passender Verdünnung ohne Wirkung auf den Sehpurpur wäre; für chemische Untersuchungen des Sehpurpurs wäre solches sehr wertvoll, da man damit in die Lage kommen könnte, ziemlich leicht verhältnismässig grosse Mengen von Sehpurpur zur Untersuchung zu erhalten.

1) H. Grenacher, Abhandl. z. vergl. Anatomie des Auges. I. Die Retina der Cephalopoden. Abhandl. der naturf, Geșellsch, zu Halle Bd. 16. 1884. 
Endlich möchte ich noch kurz über einzelne Beobachtungen berichten, die ich leider nicht genügend häufig wiederholen konnte, um weitergehende Schlüsse daraus zu ziehen, die aber doch einer kurzen Mitteilung wert sein dürften. Im Hinblicke auf $\mathrm{K} u ̈ h n e^{\prime} \mathrm{s}$ Angabe, dass an getrockneten Netzhäuten die Bleichung des Sehpurpurs ausserordentlich verlangsamt wird, versuchte ich einige rote Loligonetzhäute im Exsikkator zu trocknen; dabei blieb aber in der Regel eine tiefdunkle oder schwarze, zur Beobachtung der Purpurfärbung nicht mehr geeignete Masse zurück. Einmal gelang es mir, eine Loligonetzhaut, die 24 Stunden im Dunkeln gelegen hatte und nur noch eine Syur feucht war, derart auf dem Objektträger auszubreiten, dass sie im durchfallenden Lichte schön purpurfarbig erschien. An der Sonne wurde diese fast trockene Farbe langsam weniger rot, nach etwa einer halben Stunde Besonnung ersehien sie orangefarbig. (Der Versuch ist nicht eindeutig, da hier auch das Pigment von Einfluss auf die Färbung des durchfallenden Lichtes war.)

$\mathrm{Zu}$ den Versuchen mit taurocholsaurem Natron bediente ich mich 2-3\% iger Lösungen eines Merck'schen, zum Teile auch eines mir von Herrn Kollegen Kobert freundlichst überlassenen Präparates, die in den von mir benutzten sebr kleinen Reagensgläschen nur einen sehr schwach gelblichen Ton zeigten.

Bringt man unter dem Mikroskop zu einem Stückchen zerzupfter frischer Dunkelnetzhaut einige Tropfen des taurocholsauren Natrons, so beobachtet man eine sofort beginnende Auflösung der Stäbchen. Mit fortschreitender Lösung sieht man immer grössere Mengen von Pigment frei werden; die Stäbchen sind zunächst noch schön rot, zum Teile unregelmässig angefressen zu sehen; weiterhin erscheinen sie farblos und verschwinden allmählich vollständig; an einem Stückchen Netzhaut, das zwei bis drei Stunden in taurocholsaurem Natron gelegen hatte, konnte ich nur noch vereinzelt kleine Inselchen von nicht gelösten Stäbchen auffinden.

Bei diesen Versuchen ist natürlich nicht mit Bestimmtheit anzugeben, ob das Ausbleichen der Stäbchen durch Licht oder etwa durch das taurocholsaure Natron bedingt ist. Dagegen deuten folgende Versuche darauf hin, dass das taurocholsaure Natron in der von mir benutzten Form keinen wesentlich schädigenden Einfluss auf die Stäbchenfärbung zu haben schien.

Wird ein Stïck Netzhaut (Loligo) im Dunkeln längere Zeit in taurocholsaurem Natron gelassen und dann mikroskopisch untersucht, 
so findet man jetzt noch grosse Mengen zum Teile stark in Zerfall begriffener Stäbchen, die noch schöne Purpurfarbe zeigen. Das zur Lösung benutzte taurocholsaure Natron zeigt nun, je nach der Konzentration, mehr oder weniger gesättigt gelbrote Farbe und erscheint durch das suspendierte Pigment getrübt, um so mehr, je stärker das Präparat geschüttelt worden war. Bei Vermeiden jeden Schüttelns erhielt ich wiederholt eine fast ganz klare, aber verhältnismässig wenig gesättigte (gelblich)rote Lösung. Die Lösung wird unter der Einwirkung des Sonnenlichtes in kurzer Zeit ein wenig mehr gelblich, zugleich etwas weniger gesättigt (blasser). In einzelnen Fällen war dieses Gelblicherwerden schon nach 10-20 Sekunden, in anderen nach weniger als einer Minute wahrnehmbar; der Farbunterschied nimmt dann in der Regel nur kurze Zeit zu, danach ändert die Lösung ibre Färbung kaum oder gar nicht mehr weiter; auch nach mehrere Stunden dauernder Einwirkung des Sonnenlichtes erscheint sie in vielen Fällen nur wenig gelblicher oder blasser als kurz nach Beginn der Besonnung. Im diffusen Lichte fand ich zuweilen nach 5-15 Minuten die Lösung etwas mehr gelblich als die dunkel gehaltene; stets bleibt sie aber noch deutlich, oft ziemlich intensiv gelbrot gefärbt. Diese leichte Änderung der roten Farbe der Lösung unter dem Einflusse des Lichtes habe ich bei öfterer Wiederholung bei verschiedenen Arten (Rossia, Loligo, Sepia, Eledone) in vielen, aber nicht allen Fällen gefunden.

Die lichtbeständige mehr gelblichrote Färbung der längere Zeit besonnten Flüssigkeit scheint zum Teile durch Lösung von Pigment, zum Teile durch Suspension von solchem, infolge der Lösung der Stäbchen freigewordenem, bedingt zu sein. Ich habe einige Versuche zur Entfernung des letzteren gemacht, deren Erfolge mich aber nicht befriedigten. Durch Filtrierpapier und durch Glaswolle ging das Pigment grossenteils hindurch. Auch einige Versuche, es durch Zentrifugieren zu entfernen, gelangen nur unvollständig. In einem Falle erhielt ich nach Schütteln einer Dunkelnetzhaut von Sepia mit taurocholsaurem Natron eine rote, trübe Flüssigkeit, die nach Zentrifugieren ziemlich klar gelblichrot erschien; ein Teil davon an die Sonne gebracht erschien nach wenigen Minuten etwas gelblicher als der dunkel gehaltene Teil. Derartige Versuche gelangen nicht bloss mit ganz frischen, sondern auch mit Augen von Tieren, die längere Zeit, zum Teile sogar zwei Tage vor der Präparation, gestorben waren. 
Der Versuch, je eine vorher belichtete und eine dunkel gehaltene frische Netzhaut in taurocholsaurem Natron zu lösen, wurde dreimal angestellt. Das eine Mal war nach 24. Stunden die Farbe beider Lösungen ungefähr die gleiche; unter dem Mikroskop erschienen die noch nicht gelösten Stäbchen der Dunkelnetzhaut noch schön (gelblich) rot, die der Hellnetzhaut waren fast farblos, schwach getblich. In einem zweiten Falle erschien nach 20 Stunden die Lösung der Hellnetzhaut mehr gelb gegenüber der mehr bläulichroten der Dunkelnetzhaut. In einem dritten Falle war die eine Netzhant (Sepia) eine Stunde der Sonne ausgesetzt worden, die andere dunkel gehalten, dann jede in (ungefähr gleich grosse Mengen) taurocholsaures Natron gebracht. Nach vier Stunden war der Farbunterschied zwischen den beiden filtrierten, rot erscheinenden Lösungen nicht nennenswert. Wurde nun aber von der Lösung der Dunkelnetzhaut ein Teil in diffuses Licht gebracht, so erschien dieser nach ca. 10-20 Minuten gelber und blasser als der dunkel gehaltene Teil der Lösung. Wenn ich eine durch längere Zeit in Formol fixierte Cephalopodennetzhaut im Dunkeln in eine $1 \%$ ige Lösung von taurocholsaurem Natron brachte, so waren auch nach 2-3 Tage dauernder Einwirkung die Stäbchen noch sehr schön zu sehen. Die Flüssigkeit hatte sich wieder schön gelbrot, z. T. tiefbraunrot gefärbt, und diese Färbung erwies sich wieder sebr lichtbeständig. (Die Abnahme der Lösungsfähigkeit der Stäbchen in taurocholsaurem Natron nach Einwirkung von Formol scheint auch für die Wirbeltiernetzhaut zu gelten; wenigstens fand ich an Netzhảuten von Fröschen und Meerschweinchen, die ich in Formol fixiert hatte, auch nach zwei Tage danernder Einwirkung von $1 \%$ taurocholsaurem Natron die Stäbchen noch schön erhalten.)

Die hier mitgeteilten Versuche mit taurocholsaurem Natron haben also insofern nicht zu dem gewünschten Ziele geführt, als stets, anscheinend infolge der Lösung eines kleineren oder grösseren Teiles des Pigmentes, stark gefärbte Flüssigkeiten erhalten wurden, deren Färbung zum grossen Teile sehr lichtbeständig war und in welchen daher eine etwa unter dem Einflusse des Lichtes erfolgende Ausbleichung gelösten Sehpurpurs nicht mit genügender Sicherheit erkannt werden konnte. Weitere Versuche, zu welchen es mir bis jetzt an Gelegenheit fehlte, werden darauf gerichtet sein müssen, entweder das Pigment in einer für den Sehpurpur unschädlichen Weise zu eliminieren (s. o.), oder aber das Lösungsmittel für die Stäbchen derart zu variieren, dass dasselbe das Netzhantpigment nicht angreift.

Von Interesse erscheint der durch unsere Beobachtungen erbrachte Nachweis, dass das taurocholsaure Natron die frischen Cephalopodenstäbchen leicht zu lösen vermag, und zwar, wie es scheint, ohne stärkere Beeinträchtigung des in ihnen enthaltenen Sehpurpurs.

Durch die hier mitgeteilten Beobachtungen ist zum ersten Male 
das Vorkommen eines dem Sehpurpur der Wirbeltiere sehr nabestehenden, hochgradig lichtempfindlichen Farbstoffes in der Netzhat eines wirbellosen Tieres nachgewiesen. Es ist sowohl für das Verständnis der Bedeutung des Sehpurpurs für die Funktionen des Organs wie auch für das des physiologiseh-chemischen Prozesses von grossem Interesse, dass bei den weit voneinander entfernten Tierformen, die hinsichtlich des Baues wie auch der Ernährung der perzipierenden Elemente so ausserordentlich verschieden sind, so ähnliche lichtempfindliche Stoffe zur Entwicklung gekommen sind.

Alle Bemühungen, einen solchen Farbstoff bei Cephalopoden oder anderen Wirbellosen aufzufinden, sind bisher vergeblich gewesen. Hen s en gibt an, im Auge von Pecte n Sehpurpur gefunden zuhaben. Er schreibt ${ }^{1}$ ): „Bei Untersuchung des frischen Auges (von Pecten Jacobaeus) vor drei Jahren fiel mir auf, dass die Stäbchenschicht sehr präzise durch ihre Färbung vor den anderen Geweben der Retina hervortrat; die Färbung war chamois, aber wenn ich mich recht erinnere, auch bisweilen rötlich, ging aber a uffallend rasch verloren. Da Boll's Untersuchungen noch nicht bekannt waren, bezog ich das Ausbleichen auf Veränderungen durch die Einflüsse der Präparation und wurde davon abgehalten, den Gegenstand weiter zu verfolgen."

In Hinblick auf diese Angaben habe ich etwa 30-40 Pectenaugen von Tieren untersucht, die ich $1-18$ Stunden, zum Teile 44 Stunden lang im Dunkeln gehalten hatte. Ein Teil der Augen wurde bei möglichst herabgesetzter und eben zur Präparation hinreichender Beleuchtung auf dem Objektträger zerlegt und dann bei verschieden starker Belichtung untersucht. Ferner wurden mehrere Augen nach der Eröffnung eine halbe Stunde und länger der direkten Sonne ausgesetzt und dann bei herabgesetzter Beleuchtung mit den dunkel gehaltenen Augen verglichen. In den meisten Fällen fand ich die Netzhäute ganz farblos, in einzelnen in dickerer Schicht schwach gelblich; diese Färbung änderte sich bei der Belichtung

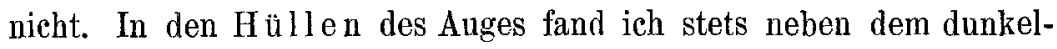
braungelben noch ein schön zinnoberrotes Pigment, das aber äusserst lichtbeständig war und auch nach langdauernder Besonnung nicht die geringste Änderung zeigte. Bei Zusatz verdünnter Salzsäure ging diese Farbe allmählich in Orange und Gelb über.

1) 1878. 1. c.

E. I'flūger, Archiv für Physiologie. Bd. 109. 
Es ist somit kein Zweifel, dass dieser Farbstoff nichts mit Sehpurpur zu tun hat. Dass mir eine Färbung der Stäbchen selbst trotz aller von mir benutzten Vorsichtsmassregeln entgangen sein könne, halte ich für ausgeschlossen.

\section{II.}

Die im Vorstehenden mitgeteilten Beobachtungen über den Sehpurpur führten mich zu Untersuchungen über die phototrope Pigmentwanderung im Cephalopodenauge. Dabei ergaben sich einige bisher nicht bekannte Tatsachen hinsichtlich des makroskopischen und mikroskopischen Verhaltens der Cephalopodennetzhaut, deren Kenntnis für das Verständnis des Folgenden erforderlich ist.

Ich schildere zunächst kurz das makroskopische Verhalten der Cephalopodennetzhaut unter verschiedenen Belichtungsverhältnissen.

Beer (1897) sagt von der Netzhaut frisch eröffueter Cephalopodenaugen allgemein, sie sei rotbraun oder schokoladefarbig, „von samtigem, fast immer vollkommen gleichmässigem Aussehen. Bei manchen Arten ist die Eintrittsstelle der Nervuli optici durch Helligkeitsunterschiede gekennzeichnet."

Hesse ${ }^{1}$ ) unterscheidet zwei Gruppen von Cephalopoden. An den in Alkohol konservierten Augen soll die eine Gruppe, zu der Rossia, Eledone, Octopus, Sepia, Sepiola gehören, tief braunschwarze Netzhäute zeigen; bei der anderen (Illex, Loligo, Todarodes, Scaeurgus) sei die Netzhaut silbergrau bis gelbgrau. Rawit $z^{2}$ ) beschrieb in der Netzhaut der frisch getöteten Sepia einen "feinen bellen Streifen, welcher genau in der Richtung des Eintrittes der Fasern des Ganglion opticum meridional verläuft"; er vermisste ihn bei Sepiola und Eledone.

Hesse fand bei den Cephalopoden der ersten Gruppe (mit dunkler Netzhaut) „überall, mit alleiniger Ausnahme von Sepiola, einen quer durch die Retina verlaufenden helleren Streifen, dessen Richtung genau der Zone entspricht, in der auf der Aussenseite die Nervenfasern das Auge verlassen, um zum Sehganglion zu gehen".

1) R. Hesse, Untersuchungen über die Organe der Lichtempfindung bei niederen Tieren. Die Retina der Cephalopoden. Zeitsehr. f. wissensch. Zool. Bd. 68 H. 3 S. 379.1900.

2) Rawitz, Zur Physiologie der Cephalopodenretina. Arch. f. Anat. w. Physiol., physiol. Abt. 1891 H. 5 u. 6. 
Hensen ${ }^{1}$ ) gab von der Netzhaut des Cephalopodenauges an, sie besitze "einen gelben Fleck, der nicht in der Mitte liegt, sondern seitlich an der verdickten Knorpelhant zu finden ist und also nur bei Verschiebung der Linse nach vorn die Achsenstrahlen bekommen kann". Beer bat wobl kleine distinkte Flecke in der Peripherie, aber auch im Zentrum gesehen, die er aber als pathologische Bildungen auffasst; einen gelben Fleck fand er nicht. Auch ich habe vergeblich nach einem solchen gesucht.

Ich habe bei sämtlichen Cephalopodenarten, die ich auf Sehpurpur untersuchte, Beobachtungen in der Weise angestellt, dass ich das makroskopische Verhalten der Netzhäute verschieden lange Zeit hell bezw. dunkel gehaltener Tiere zunächst frisch, unmittelbar nach der Enukleation, feststellte, weiterhin die oft beträchtlichen Veränderungen ermittelte, die in dem Aussehen der Netzhäute unter dem Einflusse der Konservierungsflüssigkeit (in der Regel $4 \%$ Formolseewasser, danach Alkohol von steigender Konzentration) eintraten. Dabei ergab sich für die verschiedenen Arten folgendes Verhalten:

Sepia officinalis. Bei Tieren, die 15,48 bis 87 Stunden lang dunkel gehalten waren, erscheint die frische Netzhaut schön dunkel purpurfarbig, ohne Andeutung von Streifen, die fixierte gleichmässig grau (Fig. 1), unten eine Spur dunkler als oben. Zuweilen ist in der Mitte ein wagerecht verlaufender Streif angedeutet. Auch bei einem nur vier Stunden dunkel gehaltenen Tiere ist die gehärtete Netzhaut noch ganz hellgrau, in der oberen Hälfte ein wenig heller als in der unteren.

Bei einem drei Stunden im Halbdunkel gehaltenen Tiere erscheint die frische Netzhaut dunkelbraun; in ihr ist ein wagerechter schwarzer Streif sehr deutlich sichtbar. Nach Formolhärtung erscheint an seiner Stelle ein sehr scharf begrenzter schmaler hellgrauer, fast weisser Streif. Das gleiche Verhalten fand ich bei einem Tiere, das erst eine Stunde lang an der Sonne, dann eine halbe Stunde dunkel gehalten war: Das frisch eröffnete Auge zeigt den schwarzen Streif, der schon, nachdem das Auge drei bis vier Minuten in Seewasser ans Helle gebracht war, sich in einen hellgrauen verwandelte. (Fig. 2 zeigt das Aussehen der fixierten Netzhaut dieses Auges.)

Bei Sepien, die in der Frühe tot oder sterbend im Bassin ge-

1) l. c. 
funden wurden, sah ich öfter die mittleren Netzhautpartien in ziemlich grosser Ausdehnung hellgrau, nach oben und unten allmählich in dunkleres Grau übergehend.

Die folgende Beobachtung zeigt, von welchen Umständen es abhängen kann, ob man einen hellen oder einen dunklen Streifen in der Netzhant findet.

Bei einer Sepia fand sich nach Besonnung durch eine Stunde ein schöner, schmaler, wie mit Lineal gezogener beller Streif in dem frisch eröffneten Auge; die obere Netzhauthälfte erschien mehr rötlich, die untere mehr bräunlich; der Streifen lag im rötlichen Gebiete. Im gehärteten Auge erschien die untere Netzhauthälfte grau, die obere rotbraun; der Streif war als hellgrane Linie deutlich zu sehen. Die Membrana hyaloidea löste sich leicht in toto ab; sie war stark pigmenthaltig und zeigte entsprechend dem Netzhautstreif eine feine hellgraue Linie; nach Ablösung der Hyaloidea erschien der Streif in der Netzhaut als dunkle Linie.

Bei einer 21/2 Stunden besonnten Sepia fand sich friseh in der braunroten Netzhaut ein tiefdunkler schwarzer Streif, der nach Formolfixierung hellgrau wurde.

Sepia elegans. Bei zwei Tieren, die $2^{1 / 2}$ bezw. 17 Stunden im Dunkeln gebalten waren, fand ich im gehärteten Auge gleichmässig graue Netzhäute mit einer sehr feinen dunkelbraumen Linie etwas nach oben von der Netzhautmitte.

Bei einem anderen $2^{1 / 2}$ Stunden dunkel gehaltenen Tiere waren die mittleren Netzhautpartien hellgrau, die peripheren etwas dunkler. Zuweilen erschien der Streif im gehärteten Auge hellgrau, in einzelnen Fällen von schmalen dunklen Bändern eingefasst.

Bei einem eine Stunde in mässig hellem Lichte gehaltenen Tiere erschien die untere Netzhauthälfte (des gehärteten Auges) braun, die obere rötlich. In der letzteren fand sich eine feine dunkle Linie.

Bei einem $1^{1 / 2}$ Stupden im Hellen (aber nicht direkter Sonne) gehaltenen Tiere war die gehärtete Netzhaut tiefdunkel, in den mittleren Teilen etwas weniger dunkel als in den seitlichen.

Bei einem Tiere, das erst 17 Stunden im Dunkeln, dann $4^{1 / 2}$ Stunden an der Sonne gehalten war, fand sich die untere Netzhautpartie in grösserer Ausdebnung fast schwarz, nach oben hin allmählich etwas weniger dunkel werdend; in diesem Teile war eine feine schwarze Linie sichtbar. 
Sepia Orbignyana. Bei einem vier Stunden dunkel gehaltenen Exemplar war die fixierte Netzhaut gleichmässig hellgrau;

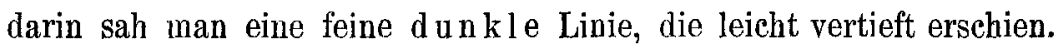

Bei einem zwei Stunden dunkel gehaltenen Tiere erschien (Fig. 3) ein kleiner, nach oben gelegener Teil der fixierten Netzhaut rotbraun, der übrige, viel grössere hellgrau; in diesem Teile, nach oben von der Mitte, sah man einen schmalen braunen Streif, in dessen Mitte wieder eine sehr feine hellgraue Linie.

Nach Besonnung während einer Stunde fand ich die frische Netzhaut oben und unten braun, in der Mitte ein helleres Band und in dessen Mitte eine feine hellgraue Linie; nach Fixieren erschien diese Linie dunkel, fast schwarz, in einem hellgrauen Bande; die peripheren Netzhautteile waren dunkler, der untere Teil wieder dunkler als der obere.

Sepiola Rondeletti. Bei Tieren, die 2-15 Stunden oder 4-6 Tage im Dunkeln gehalten waren, fand ich die fixierte Netzhaut in der Mitte hellgrau, nach unten hin allmählich dunkler werdend. Von Streifen war bei diesen Dunkeltieren nichts zu sehen.

Bei einem Tiere, das eine Stunde dunkel gehalten war, erschien die fixierte Netzhaut in den oberen zwei Dritteln rötlichbraun, im unteren Drittel hellgrau; im roten Teile war ein verwaschener grauer Streif sichtbar.

Bei einem Tiere, das erst in Hellen, dann eine Stunde im Dunkeln gehalten war, erschien die fixierte Netzhaut ziemlich gleichmässig hell braun und ohne Streifen. Die Netzhaut von an trüben Tagen getöteten Tieren war oft dunkelbraun und ohne Streif, ebenso jene von Tieren, die stundenlang an der Sonne gehalten waren.

Bei zwei Sepiolen, die $2^{1} / 2$ Stunden an der Sonne gehalten waren, fand ich in den mittleren Netzhautpartien des gehärteten Auges verwaschene helle Streifen in dunkler Umgebung. Bei einem fünf Stunden besonnten Tiere erschien die Netzhaut oben rotbraun, unten aschgrau; die Grenze zwischen beiden Zonen war scharf, von Streif nichts zu sehen.

Bei einem $4 \frac{1}{2}$ Stunden dunkel gebaltenen Tiere fanden sich (Fig. 4) die mittleren Netzhautteile braun, die oberen und unteren wesentlich heller, zum Teile grau (pathologisch?). Bei einem anderen, ebensolange dunkel gebaltenen Tiere waren die mittleren Netzhautpartien heller als die oberen und unteren.

Bei einem sechs Stunden an der Sonne gehaltenen Tiere fand 
sich eine mittlere, bandartige hellgraue Zone; nach oben und unten davon war die Netzhaut dunkler; die Hyaloidea löste sich an den dunklen Stellen stark pigmenthaltig ab, entsprechend dem Bande war sie anscheinend pigmentfrei und ganz undurchsichtig.

Eledone moscata. Nach zwei Stunden Dunkelaufenthalt erschien die fixierte Netzhaut schon ganz grauweiss, unten ein wenig dunkler als oben. Ähnlich sah sie bei einem 44 Stunden dunkel gehaltenen Tiere aus. In einem Falle dagegen war die (fixierte) Netzhaut nach 20 Stunden Dunkelaufenthalt noch dunkelrotbraun.

Bei einer Eledone, die erst eine Stunde dunkel gehalten, dann eine Stunde in mässig helles Licht (nicht direkte Sonne) gebracht war, sah ich an der frisehen Netzhaut einen schönen schwarzen Streif in dunkelbrauner Umgebung. Es war zunächst kein Unterschied zwischen unterer und oberer Hälfte zu sehen. Nachdem die Netzhaut wenige Minuten im Hellen in Seewasser gelegen hatte, erschien sie in ihrem unteren Drittel stumpfbraun, in den oberen zwei Dritteln mehr rötlich und heller; in diesem Teile lag der dunkle Streif; nach Fixierung in Formol fand sich der erwähnte Farbunterschied zwischen unterer und oberer Hälfte nicht mebr, der dunkle Streif war aber noch schön zu sehen.

Bei einem Tiere, das erst drei Tage dunkel, dann fünf Minuten an der Sonne gehalten worden war, fand sich frisch ein schmaler tiefdunkler, scharf begrenzter Streif, der nach einer Viertelstunde einen grauen Schimmer zeigte. Das fixierte Auge war von graulicher, leicht spontan und ganz pigmentfrei sich ablösender Hyaloidea überzogen. Nach Entfernung derselben ersehien die Netzhaut oben und unten grau; in der Mitte war ein schwarzer Streif sichtbar. Auch bei längerer Besonnung fand sich am frischen und oft auch am fixierten Auge der schwarze Streif; Fig. 5 zeigt die (gehärtete) Netzhaut eines im Hellen entbluteten, Fig. 6 die eines drei Stunden an der Sonne gehaltenen Tieres. Die Hellnetzhaut erscheint im Gegensatze zur Netzhaut dunkel gehaltener Tiere dunkelbraun, in der Regel (wie auch am frischen Auge) in der unteren Hälfte mehr bräunlich, in der oberen mehr rötlich. Zuweilen sieht man in den mittleren Partien einen etwas helleren Streif, umgeben von schmalen dunklen Bändern.

So fand ich bei einem Tiere, das erst 18 Stunden im Dunklen, dann fünf Minuten an der Sonne gehalten worden war, schon im 
frischen Auge einen feinen weissen Streif und die Netzhaut oben deutlich heller als unten; im fixierten Auge war wieder ein hellgrauer Streif sichtbar, die Netzhaut nach Entfernung der stark pigmenthaltigen Hyaloidea schön rotbraun.

(Der Versuch scheint zu zeigen, dass schon nach Besonnung während fünf Minuten ein ausgiebiges Vorwandern des Pigments erfolgt war; s. u.)

Auch bei einem Auge, das nach 21 Stunden Dunkelaufenthalt 20 Minuten bei mässig hellem Lichte gehalten worden war, fand ich die Netzhaut graubraun, in ihr einen schwarzen, nicht ganz scharf begrenzten Streif. Hier löste sich aber die Membrana hyaloidea ohne Pigment ab. Auch nach ihrer Entfernung war ein sehwarzer Streif in dunkelbrauner Netzhaut zu sehen.

Octopus vulgaris und macropus. Die Netzhäute von Tieren, die 18 bezw. 40 Stunden dunkel gehalten waren, erschienen frisch dunkelrotbraun; von Streifen war nichts zu sehen. Die im Hellen oder an der Sonne gehaltenen Tiere zeigten oft, doch nicht immer, im frischen Zustande einen dunklen oder schwarzen Streifen in brauner Netzhaut; auch hier war häufig die untere Netzhautbälfte mehr braun, die obere etwas mehr rötlich; im gehärteten Auge war die Netzhaut dunkelbraun, zuweilen unten deutlich dunkler als oben, der Streif ersehien oft hellgrau auf dem dunkleren Grunde.

Die in Fig. 7 abgebildete Netzbaut stammt von einem Tiere, das sechs Stunden teils dem diffusen Tageslichte, teils direkter Sonne ausgesetzt war. Die frische Netzhaut des anderen Auges dieses Tieres war gleichmässig braun ohne Andeutung von Streifen. In Formol wurde sie oben heller braun als unten. (Es ist nicht ausgeschlossen, dass das abgebildete Auge einem abnormen Zustande entspricht.)

Octopus Defilippi. Bei drei Tieren, die 18 bezw. 48 und 90 Stunden dunkel gehalten waren, erschienen die frischen Netzbäute dunkelrotbraun, ohne Streif, die fixierten hellgrau (Fig. 8), nach der Peripherie oben und unten etwas dunkler werdend. In der hellen mittleren Partie war ein feinster, wie mit Lineal gezogener dunkelbrauner Streif zu sehen. Dieser war in ähnlicher Weise an den frischen Netzhäuten von Tieren sichtbar, die $2^{1 / 2}$ Stunden im Dunkeln gewesen waren, ferner bei einem solchen, das erst zehn Minuten der Sonne ausgesetzt, dann $1^{1 / 2}$ Stunden dunkel gehalten, endlich auch bei Tieren, die eine bis zwei Stunden der Sonne ausgesetzt gewesen waren. 
Bei einem im Hellen getöteten Tiere war an der frischen Netzhaut der Streif schön schwarz, wurde aber in Formol bald grau. Im gehärteten Auge erschien die Netzhaut unten braun, oben mehr rötlichbraun, bei einem zwei Stunden besonnten Tiere dunkelbraun.

Loligo vulgaris und Loligo marmorae. Das frisch eröffnete Ange zeigt bei Hell- wie Dunkeltieren eine sehr dunkle, fast schwarze Netzhaut; an Dunkelaugen ist in der Regel zwischen der oberen und unteren Hälfte kein deutlicher Unterschied zu sehen, zuweilen erscheint die untere mehr bräunlich, die obere mehr rötlich. An Augen, die bis zum Tode im Hellen gehalten waren, erschien die frische Netzhaut zuweilen gleichmässig dunkel; häufig sieht man aber einen deutlichen Unterschied zwischen oberer und unterer Netzhauthälfte: die kleine obere Hälfte erscheint heller, rötlichbraun, während die grössere untere Hälfte dunkler, fast schwarz aussieht. Der Übergang von der einen in die andere Hälfte ist in der Regel ein allmählicher, in einzelnen Fällen stellt er sich aber als ziemlich scharfe horizontale Linie dar; ein hellerer mittlerer Streif ist an frisch eröffneten Augen im allgemeinen nicht zu sehen.

An den gehärteten Augen sind wesentlich grössere Unterschiede zwischen Hell- und Dunkelaugen wahrzunehmen:

Augen, die vor der Enukleation zwei bis fünf Stunden dunkel gehalten waren, zeigen eine gleichmässig bellgraue Netzhaut ohne Andeutung einer helleren Partie in der Mitte. Aber schon an einem Auge, das vor dem Tode eine Stunde lang im Halbdunkel gehalten war, fand ich die gehürtete Netzhaut dunkler grau, in der unteren Hälfte dunkler als in der oberen. Ein Auge, das eine Stunde bei wechselnder Bewölkung im Hellen gehalten war, zeigte nach Fixierung einen schönen, ca, $4 \mathrm{~mm}$ breiten hellgrauen Streifen in der Netzhautmitte; bei zunehmender Belichtung wird dieser Streif immer schmaler und kann schliesslich vollständig verschwinden; nach genügend langer Besonnung erscheint die gehärtete Netzhaut gleichmässig tief dunkel, fast schwarz; so fand ich sie z. B. bei einer Loligo marmorae, die $21 / 2$ Stunden der Sonne ausgesetzt war.

Fig. 9 zeigt ein Auge von Loligo vulgaris, die eine Stunde im Dunkeln, danach eine Stunde an der Sonne gehalten worden war, Fig. 10 das Auge einer im Hellen gestorbenen Loligo marmorae. Fig. 11 und 12 geben die Netzhaut von Loligo vulgaris nach verschieden langer Lichtwirkung wieder.

Rossia macrosoma. Bei einem durch fünf Tage be- 
ständig im Dunkeln gehaltenen Tiere erschien die frische Netzhaut dunkelrot, die gebärtete zeigte einen schönen, breiten, nicht scharf begrenzten, an einzelnen Stellen wie doppelt erscheinenden grauen Streifen, der eine heller graue obere Netzhauthälfte von einer viel dunkler grauen unteren trennte.

Dagegen zeigte die Netzhaut eines anderen Tieres, das gleich lang dunkel gebalten, dann aber zehn Minuten der Sonne ausgesetzt worden war, frisch eine schokoladenbraune Farbe und ca. $2 \mathrm{~mm}$ breiten hellen Streif mit Einlagerung von unregelmässigen, viel helleren Stellen. Das gehärtete Auge bot das in Fig. 13 wiedergegebene Bild: Die peripheren Netzhautteile sind oben und unten dunkelbraunrot, in der Mitte ist ein feiner heller Streif sichtbar und zu beiden Seiten von ihm unregelmässig gesprenkelte helle Stellen (zum Teile pathologisch?).

An der gehärteten Netzhaut eines vier Stunden dunkel gehaltenen Tieres fand sich eine feine horizontale hellgraue Linie, die eine grössere, dunklere untere von einer kleineren helleren oberen Partie trennte.

Bei einem Tiere, das $2^{1 / 2}$ Stunden der Sonne ausgesetzt war, erschien die frische Netzhaut hellschokoladenbraun, und es war kein Unterschied zwischen oberer und unterer Hälfte zu sehen. Durch die Netzhaut zog ein feiner schmaler, tiefschwarzer Streif; im gehärteten Auge dagegen erschien derselbe hellgrau. Das gleiche Verhalten fand ich bei einem zweiten, längere Zeit besonnten Auge; die untere Netzhauthälfte erschien viel dunkler als die obere.

Scaeurgus (unicirrus und tetracirrus). Bei einem 24 Stunden dunkel gehaltenen Tiere ersehien die frische Netzhaut schön rotbraun, nach der Härtung hellgrau; dieses Grau war in den mittleren Netzhautteilen etwas heller als oben und unten.

Bei einem Tiere, das an einem trüben Regentage $3^{1 / 2}$ Stunden im Hellen gehalten war, erschien die frische Netzhaut stumpf schokoladenbraun mit feinem dunklem Streif; auch nach der Fixierung war sie graubraun, die untere Hälfte deutlich dunkler als die obere; die Grenze zwischen beiden war durch einen scharfen dunklen Streif gebildet.

Ein ähnliches Bild fand sich bei einem Scaeurgus unicirrus, der $3^{1 / 2}$ Stunden der Sonne ausgesetzt war, dabei aber seine spaltförmigen Pupillen vollständig geschlossen hatte; der schwarze Streif in der Netzhaut erschien hier scharf, wie mit dem Lineal gezogen (Fig. 14). 
Illex Coindetti. Von Illex Coindetti standen mir nur vier lebende Exemplare zur Verfügung; von diesen waren drei anscheinend schon sehr matt, nur eines frisch und lebhaft.

Bei den drei ersten erschien die Netzhaut tıisch bräunlichrot, im gehärteten Auge hellgrau, obschon die Tiere vor der Enukleation noch einige Minuten, eines sogar noch $1 \frac{1 / 2}{2}$ Stunden lang der Sonne ausgesetzt waren. Das vierte, sehr lebhafte Tier dagegen zeigte frisch eine gleichmässig rotbraune Netzhaut, in deren Mitte ein feiner gerader, aber nicht sehr deutlicher Streif sichtbar war. Im gehärteten Auge erschien die Netzhant noch dunkler als im frisch eröffneten; auf dem Querschnitte war sie glejchmässig bellbraun, unten etwas dunkler als oben. Es war also (s. u.) nur bei diesem einen, noch in genügend frischem Zustande besonnten Tiere eine Pigmentwanderung vor sich gegangen.

Todaropsis Veranyi. Bei einem zwei Stunden im Halbdunkel gehaltenen Tiere erschien die frische Netzhaut rotbraun, die gehärtete in der oberen Hälfte mehr grau, in der unteren mehr brauu, auf dem Querschnitte oben hellgrau, unten tiefbraunrot. Bei einem moribunden Tiere, das noch zehn Minuten an die Sonne gebracht worden war, war die frische Netzhaut gleichmässig schokoladenbraun, ohne Streif, auf dem Querschnitte farblos. Bei einem anderen, woch sehr frischen Exemplare, das drei Stunden teils bei Sonne, teils bei bedecktem Himmel dem Lichte ausgesetzt war, erschien auch die gehärtete Netzhaut dunkelrotbraun, in der oberen Hälfte vielleicht eine Spur heller als in der unteren, auch auf dem Schnitte; die Membrana hyaloidea löste sich stark pigmenthaltig ab. Auch bei einem zwei Stunden besonnten Tiere erschien die Netzhaut auf dem Querschnitte grossenteils rotbraun. (Bei verschiedenen Exemplaren von Todaropsis waren anscheinend krankhafte Veränderungen in der Netzhaut vorhanden. Einnal fand ich das folgende Bild, von dem ich nicht sagen kann, ob es normal oder pathologisch ist: Bei einem Tiere, das 24 Stunden, nachdem ich es ins Dunkelbassin gesetzt hatte, tot gefunden worden war, zeigte die gehärtete Netzhaut (Fig. 15) hellgraue Farbe und in der Mitte einen ca. $4 \mathrm{~mm}$ breiten viel dunkleren und rötlichen Streifen mit ziemlich scharfer Grenze.)

Die hier kurz zusammengestellten Beobachtungen zeigen, dass bei allen von mir untersuchten Cephalopodenarten, den als pelagische 
wie den als litorale bezeichneten, die Farbe der frischen Netzhaut braun bis braunrötlich erscheint, und dass die der gehärteten Netzhaut wesentlich durch die Belichtung des Auges während der letzten Lebensstunden bestimmt wird. Es ist bei fast allen von mir untersuchten Cephalopodenarten die fixierte Netzhaut nach kürzerem oder längerem Dunkelaufenthalte melir oder weniger gleichmässig hellgrau. Nach vorausgegangener Belichtung erscheinen alle Netzhäute mehr oder weniger braun bis schwarz infolge Vorwanderns des Pigments bis zur vitralen Netzhautoberflä.che. Die Schnelligkeit, mit welcher das Pigment wandert, ist bei verschiedenen Arten offenbar ziemlich verschieden, so dass bei manchen - Octopus, Eledone, Sepia - schon nach kurzem Aufenthalte in mässig hellem Lichte die Netzhaut braun erscheint, während bei anderen, Loligo, Scaeurgus, Illex, Todaropsis, im allgemeinen erst nach Einwirkung von hellerem bezw. Sonnenlichte eine braun gefärbte Netzhaut gefunden wird. In der Regel erfolgt die Wanderung in der Weise, dass zunächst das Pigment in den peripberen Partien der unteren Netzhautteile vorrückt, dann jenes in den peripheren Partien der oberen Netzhautteile, so dass nach einem bestimmten Belichtungsgrade die mittleren Netzhautpartien im gehärteten Auge als mehr oder weniger verwaschener hellgrauer Streif in dunkler Umgebung erscheinen können.

Anf die bisher nicht genügend berücksichtigte Tatsache des Einflusses vorausgegangener Belichtung auf die Farbe der fixierten. Netzhaut sind wohl verschiedene scheinbare Widersprüche in der Literatur zurückzuführen; so hat Grenacher angegeben, dass bei Sepia und Loligo nur die „äussere" Pigmentzone im Sockel der Stäbchen erhalten sei, was für Sepia von Rawitz und von Hesse als irrtümlich bezeichnet wird; tatsächlich braucht aber ein Irrtum Grenacher's nicht vorzuliegen; denn bei einer z. B. am frähen Morgen etwa noch im Halbdunkel getöteten Sepia kann, wie wir sahen, sehr wohl das von Grenacher geschilderte Verhalten gefunden werden. Andrerseits fanden wir sogar bei Loligo die Netzhant grösstenteils schwarz und selbst im Gebiete des Streifs tiefdunkelgrau, wenn das Tier einige Zeit dem Sonnenlichte ausgesctzt gewesen war.

Der etwas nach oben von der Netzhautmitte sichtbare Streif erscheint nicht, wie angegeben wird, stets hell, sondern in einer Reihe von Fällen tiefdunkel, fast schwarz in weniger dunkler Ungebung. Er findet sich auch keineswegs nur bei den litoralen Formen (Hesse), sondern kann in ganz ähnlicher Weise auch bei den von Hesse als pelagische bezeichneten Formen (Scaeurgus und Rossia) deutlich sichtbar sein, während er andererseits bei solchen Sepien, die dunkel gehalten waren, ganz oder fast ganz unsichtbar 
sein kann. Danach erscheint weder eine scharfe Scheidung von Cephalopodenarten mit beller und solchen mit dunkler Netzhaut noch die Einteilung in Arten mit und ohne Streif angängig. Ob der Streif heller oder dunkler erscheint als die Umgebung, längt nicht ausschliesslich von der jeweiligen Stellung des Pigmentes, sondern jedenfalls zum Teile von ganz andersartigen Umständen ab.

Die Angabe (Beer, Rawitz), dass dieser Streif genau dem Eintritte der Nervuli optici entspreche, ist nicht zutreffend. Ich habe die einschlägigen Verbältnisse bei verschiedenen Arten genauer untersucht und gefunden, dass einmal die Richtung des Streifs durchaus nicht jener des Sehnerveneintrittes entspricht, sondern in vielen Fällen mit ihr einen mehr oder weniger spitzen Win̛kel bildet; zweitens kann die Haupteintrittsstelle der Sehnerven mehrere Millimeter weit von der Stelle des Streifs entfernt sein, und drittens pflegt die Zone, innerbalb deren die Sehnervenfasern die Sclera durchsetzen, viel breiter zu sein als der helle Streif in der Netzhaut. Lage und Anordnung des letzteren ist offenbar wesentlich oder ausschliesslich durch die Sehbedürfnisse des Tieres bestimmt, nicht aber durch anatomische Beziehungen zu dem Sehnerven und von diesem in ziemlich weiten Grenzen unabhängig.

Die anatomischen Verhältnisse der Netzhaut im Streifengebiete werden im folgenden Abschnitte genauer zu erörtern sein. Zunächst sei noch kurz das makroskopisehe Verhalten der Membrana hyaloidea in verschiedenen hell bezw. dunkel gehaltenen Augen besprochen.

Die Membrana hyaloidea löst sich bei den in Formol fixierten und in Alkohol nachgehärteten Augen häufig spontan, in vielen Fällen bei schwachem Ziehen mit der Pinzette leicht, in einigen Fällen schwerer von der Unterlage ab. Auch hier zeigt sich der Einfluss der Belichtung auf das Aussehen der abgezogenen Membran in auffälliger Weise: An den Augen dunkel gehaltener Tiere löste sich die Membran in der grossen Mehrzahl der Fälle völlig pigmentfrei von der Netzhant. Dies war z. B. meist der Fall bei Sepia, Octopus, Eledone. An belichteten Augen dagegen findet sich verhältnismässig häufig (aber nicht ausnahmslos) die Hyaloidea, auch wenn sie sich spontan von der Netzhaut löste, mehr oder weniger stark pigmenthaltig, und zwar ist das Pigment auf ihr in zahlreichen, meist rundlichen Kugeln angehäuft. Solches liess sich auch für belichtete Loligoaugen feststellen. An Dunkelaugen fand ich gelegent- 
lich (jedoch nicht eben häufig) auf der Membran farblose, mit Eosin sich schwach rosa färbende Kugeln aufliegend.

Entsprechend dem Streif in der Netzhaut zeigte die Membran kein ganz konstantes Verhalten: In manchen belichteten Augen fand ich die Hyaloidea im allgemeinen stark pigmenthaltig und nur entsprechend dem Streifen pigmentfrei; in anderen Fällen war sie umgekehrt nur dem Streif entsprechend pigmenthaltig oder doch hier deutlich stärker pigmenthaltig als in der Umgebung; in wieder anderen Fällen fand sich entsprechend dem Streif auf der Hyaloidea eine graue Partie, die gelegentlich wie eine Auflagerung aussah, zuweilen aber in der Membran zu liegen schien.

Aus diesen Untersuchungen, die ich hauptsächlich an Loligo, Eledone, Sepia und Octopus angestellt habe, geht also hervor, dass die Wanderung des Pigmentes über die innere Oberfläche der Netzhaut hinaus erfolgt, so dass dieses bier in sehr innige Berührung mit der Hyaloidea kommt und sich bei der Konservierung oft leichter von der Netzhaut als von jenem Häutchen trennt; das verschiedene Aussehen des Netzhautstreifs im frischen und im konservierten Auge bei den früher besprochenen Fällen kann wenigstens zu einem Teile auf die geschilderten Eigentümlichkeiten der Membran entsprechend dem Streif bezogen werden.

\section{III.}

Bei der mikroskopischen Untersuchung der im Vorstehenden besprochenen Cephalopodennetzhäute ergaben sich einige interessante Befunde, die hier im Zusammenhange mit dem Verhalten des Pigmentes unter dem Einflusse des Lichtes geschildert werden sollen.

Genauere Untersuchungen über das anatomische Verhalten der Netzhäute in den verschiedenen Regionen des Auges fehlten bisher. In der Literatur finde ich nur eine Angabe Hensen's aus dem Jahre 1865 (nach Untersuchungen an Eledone, Sepia, Sepiola, Loligo), wonach die Stäbchen „gestreckte parallelwandige, an beiden Enden abgestumpfte Gebilde sind, die an der Peripherie kürzer und dicker, am Zentrum feiner und länger gefunden wurden. Bei Eledone sind sie im Zentrum $0,26 \mathrm{~mm}$ lang, $0,003 \mathrm{~mm}$ breit, an der Peripherie $0,06 \mathrm{~mm}$ lang, $0,006 \mathrm{~mm}$ breit"...

Dagegen betont unter den neueren Forschern Hess e gelegentlich seiner Zäblungen der Netzhautelemente (s. u.) ausdrücklich, dass die Verteilung derselben eine sehr gleichmässige, die Zählung 
daher sehr einfach sei, und Chun gibt bei seiner interessanten Beschreibung der Augen von Tiefseecephalopoden an, das normale Cephalopodenauge sei u. a. durch eine gleichmässig dicke Netzhaut charakterisiert.

Ich hatte schon früher in einer kurzen Notiz angedentet, dass bei manchen Cephalopodenarten die Stäbchen entsprechend dem "Streifen" sich wesentlich anders verhalten als in den benachbarten Partien; dies lässt sich leicht auf Schnitten nachweisen, die senkrecht zur Streifenrichtung geführt sind. Bei verschiedenen Arten findet man hier wesentlich verschiedenes Verhalten. Aus der folgenden Darstellung geht hervor, dass bei einer Reihe von Cephalopoden, und zwar bei "pelagischen" wie bei "litoralen", sich eine schmale, mittlere, von den benachbarten oft ziemlich scharf abgegrenzte Netzhautzone findet, innerhalb deren die Stäbchen viel schmäler und länger sind als in der übrigen Netzhaut. Auf einem senkrecht durch die Streifengegend geführten Schnitte erseheint schon makroskopiseh die Netzhaut entsprechend dem Streif deutlich, oft beträchtlich dicker als in den übrigen Teilen. Auch die Limitanszellen und die Kernregion zeigen hier besonderes Verhalten, und auch die Pigmentwanderung erfolgt innerhalb des Streifs in wesentlich anderer Weise als in der Nachbarschaft. Es unterliegt wohl keinem Zweifel, dass es sich hier um einen Streif des deutlichsten Sehens handelt. Bei einer zweiten Gruppe von Cephalopoden, der wiederum sowohl "pelagische" wie "litorale" Formen angehören (z. B. Sepiola, Loligo, Todaropsis), finde ich jene mittlere Zone der langen schmalen Stäbchen nicht; die Netzhaut erscheint auf dem Schnitte überall angenähert gleich dick, bei Loligo vielleicht peripher nach oben etwas dicker als unten, bei Sepiola umgekehrt unten deutlich dicker als oben. Dagegen findet auch bei diesen Arten die Pigmentwanderung in der mittleren Netzhautzone in etwas anderer Weise statt als peripher oben und unten.

Über diese Pigmentwanderung lagen bisher nur folgende Beobachtungen vor:

Rawitz sah zuerst bei Eledone, Sepia, Sepiola und Octopus eine Änderung der Pigmentstellung unter dem Einflusse langdauernder Verdunkelung. Er schreibt: „Die beschriebene Pigmentverteilung (sc. Innenstellung des Pigmentes mit Ausnahme der Stelle des bellen Streifs; siehe weiter unten), finde ich, wenn die genannten Tiere unter normalen Belichtungsverhältnissen gelebt haben." „Hat man 
die Tiere im Dunkelzimmer gehalten, so erkennt man an feinen Längsschnitten durch die Retina nach 24 Stunden und besser noch nach 48 Stunden folgendes: Das Pigment hat sich vom inneren Ende der Rhabdomregion völlig zurückgezogen, meist so weit, dass nur noch das äussere, $d$. h. der Sockelregion benachbarte Viertel Pigment enthält .... Interessant ist dabei folgende Beobachtung. Hatten die Eledone - und nur an dieser Spezies sind die Versuche mit farbigem Lichte ausgeführt worlen - eine solche Stellung angenommen, dass nur ein Teil jedes Anges belichtet, der andere im Dunkeln geblieben ist, so zeigt sich auch im mikroskopischen Präparate die Pigmentverteilung in dem belichtet gebliebenen Abschnitte der Netzhaut wie in der normalen; ich konnte also in derselben Retina und in demselben Schnitte beide Modi der Pigmentierung konstatieren." Hesse beobachtete das Ergebnis der Pigmentverschiebung an der Retina einer Sepia, die er zwei Tage in der Dunkelkammer hielt. Ob bei den von Hesse als "pelagische" aufgeführten Formen "mit hellgrauer Netzhaut" überhaupt phototrope Pigmentverschiebungen vorkommen, war bisher nicht untersucht.

Die erwähnten Beobachtungen bei sehr lange dauernder Verdunklung sind wohl an sich sehr wertvoll, können uns aber über die physiologische Bedeutung der Pigmentwanderung nicht genügend orientieren; denn unter gewöhnlichen Verhältnissen dauern Belichtung und Verdunkelung im allgemeinen nicht mehr als etwa 12 Stunden, und eine Pigmentverschiebung kann physiologisch nur dann von Bedeutung sein, wenn sie verhältnismässig rasch erfolgt.

Die ersten Angaben über eine Lageveränderung des Pigmentes in der Cephalopodennetzhaut machte wohl $\mathrm{Babuchin}{ }^{1}$ ), ohne aber die Abhängigkeit derselben von der Lichteinwirkung zu erkennen. Er sagt: „Obwohl die Stäbchen der Sepia beinahe beständig von einer Schicht Pigment überdeckt sind, so stiess ich später dennoch auf Exemplare, wo diese Schicht gar nicht vorhanden war. Dieselbe Erscheinung wiederholte sich auch bei Loligo. Der Grund liegt aber darin, dass die Pigmentsubstanz einer Dislokation fähig ist und aus der einen Abteilung der Zelle in eine andere übergehen kann ...."

Bei Hensen finde ich folgende Angaben: „Es (sc. das Pigment) bildet eine nicht unbeträchtliche Lage, auf welcher die Stäbchen ruhen; auch erstreckt es sich in die Stäbchenlage hinein und erfüllt bei Loligo und Eledone die Spitzen wiederum dichter; übrigens wird es wohl anch hier eine gewisse Beweglichkeit besitzen und also Lagenveränderungen eingehen können. Es ist mir sogar höchst

1) Babuchin, Vergleichend-histol. Studien. I. Über den Bau der Cephalopodenretina. Würzburger naturw. Zeitschr. Bd. 5. 1869. 
wahrscheinlich, dass eine so dichte Ansammlung, wie sie sich gewöhnlich bei Eledone an den inneren Enden findet, nur künstlich durch das Hervorquellen der Tropfen am freien. Ende, die mit einer Verschiebung des Pigments verbunden war, entstanden ist, wenigstens findet man hin und wieder Stellen, wo das Pigment gleichmässiger durch die ganze Schicht verteilt ist."

Ferner gehört wohl auch eine Angabe Max Schultze's hierher, der die tiefste Pigmentierung, übereinstimmend mit Hensen und anderen, bei Octopus vulgaris beobachtet bat, "doch durchaus nicht bei allen Exemplaren gleichmässig, auch nicht an allen Stellen der Retina".

Über das Verhalten des Pigmentes in dem Gabiete des Netzhautstreifs machte Rawitz folgende Angaben. "A nf einem dünnen Längsschnitte durch die gehärtete Retina, welcher den hellen Streif senkrecht kreuzt, erkennt man, dass entsprechend demselben die innere Pigmentanhäufung fehlt. Es reichen die von der äusseren Pigmentanhäufung nach der inneren, freien Fläche der Retina strebenden Pigmentzüge nicht bis zum inneren Ende der Retinazellen, sondern hören vor demselben auf, so dass die innerste Partie der Rhabdomregion ungefähr in 1/5 ihrer Ausdehnung pigmentfrei ist. Im Zentrum des hellen Streifs reicht das Pigment am wenigsten weit nach innen; nach den Seiten zu nähert es sich allmählich dem inneren Ende der Retinazellen, um peripher von dem Streif die innere knöpfchenförmige Pigmentanhäufung zu bilden" . . . .

Die folgende Darstellung enthält einen kurzen Überblick über die wesentlichen Ergebnisse meiner Untersuchungen an verschiedenen Cephalopodenaugen bei wechselnden Belichtungsverhältnissen.

Sepia officinalis. Die Netzhaut wird in einem horizontalen, streifenförmigen, etwas nach oben von der Netzhautmitte gelegenen Gebiete auf dem Schnitte beträchtlich dicker gefunden als in den übrigen Teilen. In diesem Gebiete sind die Stäbchen wesentlich schmaler, und dementsprechend stehen die zugehörigen Kerne viel dichter als in der Nachbarschaft. Ich habe bei mehreren Tieren ziemlich übereinstimmend folgende Zahlen gefunden: Stäbchenlänge im Streifengebiete $0,21 \mathrm{~mm}$, etwas seitlich davon $0,14 \mathrm{~mm}$. Im Streifengebiete zählte ich auf einer Strecke von $1 \mathrm{~mm}$ ca. 1000 Kerne, etwas seitlich davon auf der gleichen Strecke nur noch 500.

Ähnliche Verhältnisse fand ich auch bei Sepia Orbignyana; entsprechend dem Streif zählte ich ca. 850 Kerne auf $1 \mathrm{~mm}$, nur $1 / 2 \mathrm{~mm}$ nach unten davon nur noch 450 und $1 \mathrm{~mm}$ weiter nur noch 280 auf einer gleich grossen Strecke. Die Kerne erscheinen in den seitlichen Netzhautteilen vorwiegend rund, dagegen in der Mitte lang- 
gestreckt, zum Teile, anscheinend infolge der dichteren Anordnung, spindelförmig. Die Limitanszellen ragen hier weit nach hinten aus der Pigmentzone vor, während sie an den Seiten fast oder ganz von Pigment verdeckt sind.

Bei einem 15 Stunden dunkel gehaltenen Tiere fand ich das Pigment nirgends in Knöpfchen innenständig; in der unteren Netzhauthälfte reichte es in feinen Linien bis dicht an das vitrale Stäbchenende und endigte hier innerhalb der Stäbchen selbst in Form von schlanken, keulenförmigen Verdickungen. Gegen die mittleren Netzhautpartien hin findet man, in ganz allmählichen Übergängen, zunächst das innere Drittel, weiter oben etwa die innere Hälfte der Stäbchen ganz frei von Pigment; noch weiter oben ist es wieder ein wenig weiter vitralwärts gelagert. Fig. 16 zeigt die Verhältnisse im Streifengebiete. Bei einem 87 Stunden dunkel gehaltenen Tiere war das Pigment vollständig auf die Stäbchenbasis zurückgezogen. In dem Streifengebiete der langen schmalen Stäbchen zeigte das hier fast ganz skleral liegende Pigment an der Stäbchenbasis sehr regelmässige Knöpfehen und Kölbchen, ganz ähnlich jenen am vitralen Fnde belichteter Augen (vgl. auch Fig. 16). Auch von ihnen gehen feine, stark geschlängelte Pigmentzüge in das Stäbcheninnere. Dieses wandernde Pigment ist, besonders deutlich in den mittleren Netzhantteilen, von dem stabilen Pigmente getrennt, das nach aussen von ersterem in grossen Mengen kolbige Zellen erfüllt, die hier oft deutlich voneinander gesondert erseheinen und häufig stark pigmentierte, zum Teile aber auch pigmentfreie Fortsätze nach aussen bis zur Limitans schicken.

Ähnlich ist bei einer nur vier Stunden dunkel gehaltenen Sepia off. fast in der ganzen Netzhaut das Pigment bereits bis auf die Basis zurückgezogen und bildet hier wieder die vorher beschriebenen Knöpfchen; die Stäbchen selbst erscheinen frei von Pigment.

Dagegen fand ich bei einer drei Stunden im Halbdunkel gehaltenen Sepia das Pigment schon durchweg innenständig (d. h. vitral), auch in der mitt]eren Zone der feinen Stäbchen dichte vitrale Knöpfchen bildend; die Knöpfehen sind hier nur viel schmaler als an den Seiten. Hinter den vitralen Knöpfchen findet man den vitralen Stäbchenabschnitt von feinen Pigmentlinien durchzogen, in der mittleren Zone der langen Stäbchen viel dichter und reichlicher als in der übrigen Netzhaut.

Bei einer Sepia, die erst eine Stunde an der Sonne, dann eine E. PfIüger, Archiv für Physiologie. Bd. 109. 
halbe Stunde dunkel gehalten worden war, und bei der ich am frischen Ange einen schön dunkelvioletten, bei der Härtung grau werdenden Streifen in der Netzhaut gefunden hatte (vgl. Fig. 2), zeigte sich das Pigment oben und unten in dichten Knöpfchen noch innenständig, dagegen entsprechend dem mittleren Streif schon so beträchtlich zurückgezogen, dass hier keine Pigmentknöpfchen mehr $\mathrm{zu}$ sehen waren.

Bei einer eine Stunde besonnten Sepia war das Pigment überall in dichten Knöpfen innenständig, auch im Streifengebiete; die Hyaloidea hatte sich überall pigmenthaltig (spontan) abgelöst. Die Stäbchen zeigten entsprechend dem Streifengebiete binter den Knöpfehen noch feine Pigmentzüge, die an den seitlichen Netzhautteilen fehlten.

Bei einer $1 \frac{1}{2}$ Stunden besonnten Sepia war überall das Pigment innenständig, und zwar so dicht, dass es ein gleichmässig schwarzes Band an der vitralen Netzhautoberfläche zu bilden schien.

Für die Pigmentverhältnisse im Gebiete des hellen Streifs hatte Rawitz bei Sepia angegeben (s. o.), dass im Zentrum des hellen Streifens das Pigment am wenigsten weit nach innen reiche und nach den Seiten zu sich allmählich dem inneren Fnde der Retinazellen nähere, um peripher von dem Streifen die innere knöpfchenförmige Pigmentanhäufung zu bilden.

Unsere Beobachtungen zeigen, dass eine derartige Angabe genau nur für einen ganz bestimmten Belichtungsgrad zutrifft, dass aber bei etwas stärkerer Belichtung das Pigment auch im Streifengebiete selbst in dichten Knöpfen innenständig gefunden wird.

Sepia elegans. Auch bei dieser kleinen Cephalopodenart fand ich etwas nach oben von der Netzhautmitte in einer Breite von ca. $1 \mathrm{~mm}$ die Stäbchen viel länger und schlauker als seitlich davon: In der Mitte war die Stäbchenlänge bei einigen von mir gemessenen Exemplaren ca. 0,14 mm, etwas seitlich nur noch $0,10 \mathrm{~mm}$. Die Zahl der Kerne betrug im Streifengebiete auf einer Strecke von $1 \mathrm{~mm}$ ca. 1600, wenig sejtlich davon nur noch 500 .

Bei einem 21/a Stunden dunkel gehaltenen Tiere war (Fig. 17) das Pigment nirgends in Knöpfchen innenständig, in dem unteren Netzhautabschnitte etwa die vitrale Hälfte der Stäbchen, oben etwa $2 / 3 \ldots 3 / 4$ dersełben ganz frei von Pigment, während sie in der mittleren Zone so gut wie ganz frei von Pigment erschienen; letzteres war bier nur an der Stäbchenbasis in dicken Kolben zu sehen, und 
zwar, ebenso wie Sepia officinalis, vom stabilen Pigmente an vielen Stellen deutlich gesondert. Besonders auffällig ist das verschiedene Verhalten der Limitanszellen innerhalb und ausserhalb des Streifengebietes: in ersterem sieht man die Limitanszellen weit nach hinten aus der Pigmentschicht hervorragen, während sie ausserhalb des Streifs grossenteils von Pigment verdeckt erscheinen.

Bei einer $1 \frac{1 / 2}{2}$ Stunden diffusem Lichte ausgesetzten Sepia fand ich (Fig. 18) das Pigment beinahe auf der ganzen Netzhaut in dicken Knöpfchen innenständig, so dass es fast ein schwarzes Band zu bilden schien. Entsprechend dem Gebiete der langen schmalen Stäbchen reichte auf einer Strecke von ca. $0,1 \mathrm{~mm}$ das Pigment nicht bis zum vitralen Stäbchenende, sondern blieb von ihm, eine flach bogenförmige Zone bildend, so weit entfernt, dass in der Mitte etwa ${ }^{1 / 8}$ des innersten Stäbchenteiles frei von Pigment erschien. Letzteres erfüllte die skleralwärts an jene bogenförmige Zone grenzenden Stäbchenteile in einer Ausdehnung von etwa $1 / 4$ der Stäbchenlänge und bildete nach den Seiten hin auf einer kurzen Strecke noch eine zweite Pigmentzone hinter den hier bereits innenständigen Knöpfchen.

Eledone moscata. Auch hier findet man wieder in einem mittleren streifenförmigen Gebiete die Stäbchen wesentlich länger und schmäler als an den Seiten, die Netzhaut auf dem Schnitte entsprechend dicker.

Bei einem sehr kleinen Exemplare, wo die Verhältnisse sich besonders leicht übersehen liessen, mass ich in der Mitte eine Stäbchenlänge von $0,11 \mathrm{~mm}$, wenig seitlich davon eine solche von $0,09 \mathrm{~mm}$. Im Streifengebiete zäblte ich auf einer Strecke von $1 \mathrm{~mm}$ 850 Kerne, wenig seitlich davon nur noch etwa die Hälfte.

Bei einem 40 Stunden dunkel gehaltenen Tiere war das Pigment in der oberen und unteren Peripherie der Netzhaut noch zum Teile in sehr feinen Knöpfehen innenständig, zum Teile in langen Zügen in der vitralen Hälfte der Stäbchenreiben sichtbar. In der mittleren Netzhautzone fanden sich keine vitralen Knöpfchen, das Pigment hatte sich so weit zurückgezogen, dass etwa $1 / 10-1 / 12$ der innersten Stäbchenteile frei von Pigment war. Letzteres war in langen Zügen sichtbar, die skleralwärts in die erwähnten basalen Pigmentkolben übergingen.

Bei einem 20 Stunden dunkel gehaltenen Exemplar (das aber einzelne anscheinend krankhafte Veränderungen zeigte), war das Pigment überall in Kuöpfchen innenständig, ausserdem fanden sich 
hier aber auch die basalen Kolben des mobilen Pigments deutlich ausgesprochen (vgl. Fig. 19, nach einem peripher gelegenen Netzhautstücke gezeichnet).

Bei einer zwei Stunden dunkel gehaltenen Eledone war das Pigment in den peripheren Netzhautteilen oben und unten grossenteils in vitralen Knöpfchen innenständig, entsprechend dem Streif waren auf einer kurzen Strecke nur vereinzelte Knöpfchen sichtbar, im übrigen etwa das innere Zehntel der Stäbchen pigmentfrei; der übrige Teil der Stäbchen zeigte lange Züge feinen Pigments.

Bei einem Exemplar, das nach einer Stunde Dunkelaufenthaltes zehn Minuten besonnt war, fand sich das Pigment bereits überall in feinen Knöpfchen innenständig, a u ch in der Streifengegend. Die Stäbchen waren allenthalben in ihrer ganzen Länge von sehr feinen Pigmentzügen durchsetzt. Entsprechend dem Streif fand sich dicht hinter den Knöpfchen noch eine ziemlich breite Zone feiner, dicht stehender Pigmentlinien. Ebenso war bei einer im Hellen durch Entbluten getöteten Eledone (Fig. 20) das Pigment überall in dichten Knöpfchen innenständig; entsprechend dem Streifen waren die Knöpfehen viel feiner und vielleicht etwas spärlicher als an der übrigen Netzhaut. Vielfach erschienen sie hier nicht rund, sondern dreieckig. Ferner sah man hier ausser den feinen Pigmentlinien zwischen den Stäbchen noch kleine rundliche Pjgmentklümpchen. (Diese Netzhaut war nicht, wie die übrigen, in Formol, sondern in Alkohol fixiert worden.) Fig. 21 zeigt die Netzhaut einer im Hellen getöteten Eledone entsprechend dem Streif und seiner nächsten Nachbarschaft.

Auch bei einer erst 21 Stunden dunkel, dann 20 Minuten im Hellen (aber nicht in direkter Sonne) gehaltenen Eledone war überall das Pigment in Knöpfehen innenständig, auch entsprechend dem Streifen.

Wir finden alșo hier (falls nicht etwa zufällig mehrere der Augen abnorm gewesen sein sollten) selbst bei langdauernder Verdunkelung das Rückwandern des Pigmentes in sehr viel geringerem Umfange als bei den anderen bisher von mir untersuchten Arten. Kurz dauernde, mässige Belichtung genügt, um a u ch im S treifengebiete das Pigment in dichten Knöpfehen an die vitrale Oberfläche der Netzhaut treten zu lassen.

Octopus Defilippi. Bei dem kleinen Octopus Defilippi fand ich stets die Netzhaut in ihren mittleren Teilen, in einer Breite von ca. $1,5 \mathrm{~mm}$, beträchtlich dicker als seitlich. Die Stäbchenlänge betrug im mittleren Gebiete ca. $0,11 \mathrm{~mm}$, etwas seitlich davon nur noch 
$0,06 \mathrm{~mm}$; im ersteren waren auf einer Strecke von $1 \mathrm{~mm}$ ca. 800 Kerne zu zählen, wenig seitlich davon nur noch etwa 420 .

Bei einem 18 Stunden dunkel gehaltenen Tiere war das Pigment in den peripheren Netzhautpartien in spärlichen Knöpfehen innenständig, hinter den Knöpfehen in langen Reiben zwischen den Stäbchen zu sehen. Gegen die mittleren Netzhautteile hin hat es sich etwas vom vitralen Ende der Stäbchen zurückgezogen, so dass etwa das innerste Zehntel derselben pigmentfrei ist. Das Pigment bildet zwischen oder innerhalb der kurzen seitlichen Stäbchen selbst noch hellbräunliche langgestreckte kolbenförmige Gebilde, dagegen an den langen, schmalen mittleren Stäbchen nur feine, aus spärlichen Körnchen gebildete Züge.

Bei einem 48 Stunden dunkel gehaltenen Tiere waren fast nirgends innenständige Knöpfehen $z u$ sehen (einige wenige fanden sich in den untersten Netzhautpartien). In den mittleren und oberen Netzhautteilen war etwa das innere Viertel der Stäbchen ganz frei von Pigment.

Bei einem Tiere, das erst 48 Stunden dunkel gehalten, dann eine Viertelstunde diffusem Lichte (aber nicht der Sonne) ausgesetzt war, fand ich das Pigment (Fig. 22) an den Seiten und in dem grössten Teile der mittleren Netzhautpartie in dichten Knöpfehen innenständig; sogar im Streifengebiete waren reichlich innenständige Knöpfchen von grosser Feinheit zu sehen. Hinter diesen bildete das Pigment einen schwarzen Saum von fast $1 / 4$ der Stäbchenlänge. Es hat also schon diese kurze Belichtung mit diffusem Lichte genügt, um eine starke vitrale Pigmentverschiebung, auch im Streifengebiete, herbeizuführen.

Bei einem eine Stunde besonnten Tiere fand ich das Pigment überall, auch an den schmalen Stäbchen des Streifengebietes, in dichten Knöpfehen ganz innenständig. Die mittleren Knöpfchen sind feiner und leichter voneinander zu sondern als die seitlichen. Hinter ihnen sieht man wieder feine Pigmentzüge, die in der Gegend der langen feinen Stäbchen in etwa ${ }^{3 / 4}$ von deren Länge sichtbar sind, während an den Seiten nur etwa die inนere Hälfte der Stäbchen Pigment führt. (Fig. 23 gibt ein Stück der spontan abgelösten Membrana hyaloidea eines belichteten Octopus mit den daran haftenden Pigmentklümpchen wieder; vgl. S. 420.)

Nach diesen Beobachtungen zieht sich das Pigment bei Octopus Defilippi im Dunkeln nur sehr langsam zurück, und auch bei lang- 
dauernder Verdunkelung nur so weit, dass etwa $1 / 4$ des vitralen Stäbchenabschnittes ganz pigmentfrei wird. Unter dem Finflusse der Belichtung wandert das Pigment rasch und ausgiebig vitralwärts vor. Sepiola Rondeletti. Auf dem Schnitte erschien die Netzhaut in der Regel in der äussersten Peripherie unten am dicksten; ihre Dicke nahm nach oben hin allmählich etwas ab; von einer dickeren mittleren Partie konnte ich in keinem Falle etwas finden; auch mikroskopisch fehlten der mittleren Zone die feinen und langen Stäbchen. Diese waren vielmebr überall ungefähr gleich lang, und auch die Kerne waren allenthalben in angenähert gleichmässiger Verteilung zu sehen; die Limitanszellen ragten überall ziemlich beträchtlich aus der Pigmentzone bervor.

Bei einem $4 \frac{1}{2}$ Stunden dunkel gehaltenen Tiere fand sich das Pigment in den oberen Netzhautteilen, bis gegen die Mitte hin, in dichten Knöpfchen innenständig. In einer mittleren Netzhautzone war es ein wenig von der vitralen Oberfläche zurückgezogen und teils in Streifen, teils in keulenartigen Bildungen in der Stäbchenschicht gelegen. In den unteren Netzhautteilen lag es wieder in dichten Knöpfchen an der vitralen Oberfläche, noch mehr peripherwärts war es zum grösseren Teile wieder etwas zurückgezogen.

Bei einem eine Stunde dunkel gehaltenen Tiere füllte das Pigment in feinen Zügen von etwa $1 / 4$ der Stäbchenlänge ungefähr die mittleren Stäbchenteile, überall ziemlich glejch weit von den Stäbchenenden entfernt bleibend. In der unteren Netzhauthälfte fanden sich spärlich innenständige Knöpfchen, hinter ihnen zum Teile, in etwa $1 / 6$ der Stäbchenlänge, feine Pigmentzüge.

Bei einem eine Stunde besonnten Tiere war das Pigment überall in feinen Knöpfchen innenständig, die Hyaloidea löste sich úberall pigmenthaltig ab. Danach wären die anatomischen Verhältnisse hier anscheinend ähnliche, wie etwa bei Loligo.

Loligo vulgaris und Loligo marmorae. Hier fand ich (ebenso wie bei Todaropsis, s. u.) die Netzhaut auf dem Schüitte an allen Stellen ungefähr gleich dick, die Stäbchen angenähert gleich lang: Bei einer grossen Loligo vulgaris waren sie zum Teile 0,18 nm, bei einer kleinen Loligo marmorae etwa $0,08 \mathrm{~mm}$ lang; dementsprechend war die Zahl der Kerne an allen Stellen angenähert gleich gross, jedenfalls fehlte eine mittlere Zone mit relativ sehr grosser Kernzahl. Die Limitanszellen ragten überall weit nach hinten vor. Uier fehlten also die anatomischen Verhältnisse, die bei den 
meisten anderen, vorher besprochenen Arten der Bildung des Streifs zugrunde lagen; trotzdem finden sich in mancher Beziehung ähnliche physiologische Verhältnisse der Pigmentwanderung wie dort.

Bei einer eine Stunde im Dunkeln gehaltenen Loligo marmorae war das Pigment vollständig auf die Stäbchenbasis zurückgezogen, die Stäbchen erschienen ganz frei von Pigment. Bei einem Tiere, das erst eine Stunde im Dunkeln, dann eine Stunde an der Sonne gehalten war, fand ich das Pigment im unteren Drittel der Netzhaut in dichten Knöpfchen innenständig, in den beiden oberen Dritteln dagegen nirgends innenständig, vielmehr in feinen Zügen zwischen den Stäbchen in deren skleraler Hälfte. Dementsprechend löste sich die Hyaloidea unten pigmenthaltig, oben pigmentlos von der Netzhaut $a b$; die Grenze zwischen beiden Netzhautzonen war ziemlich scharf.

Bei einer im Hellen gestorbenen Loligo marmorae fand sich gleichfalls das Pigment in den oberen zwei Dritteln fast ganz skleral, im unteren Drittel dagegen rejehte es bis nahe zur inneren Oberfläche der Netzhaut, liess nur eine kleine Strecke der vitralen Stäbchenenden frei und bildete zwischen den Stäbchen selbst kleine Keulen.

Nach einer Stunde Besonnung fand ich bei einer Loligo marmorae das Pigment in der oberen und unteren Netzbauthälfte bis dicht an das vitrale Stäbchenende vorgewandert, die innere Stäbchenhälfte in langen Zügen ausfüllend, zum Teile Knöpfchen bildend. Auch hier war das vitrale Ende der Pigmentzüge zwischen den Stäbchen beträchtlich dicker als der übrige Teil. In den mittleren Netzhautpartien dagegen fand sich das Pigment fast ganz auf den skleralen Stäbchenabschnitt beschränkt. Ähnliches Verhalten fand ich bei einer Loligo vulgaris, die erst eine Stunde dunkel, dann eine Stunde an der Sonne gehalten war.

Rossia macrosoma. Auf einer streifenförmigen mittleren Netzhautzone von ungefäbr $1,5 \mathrm{~mm}$ Breite sind die Stäbchen länger und feiner (vgl. Fig. 24) als oben und unten davon. (In der Mitte ca. $0,23 \mathrm{~mm}$, wenig oben und unten davon nur $0,16 \mathrm{~mm}$ bei einigen von mir gemessenen Exemplaren. Die Zahl der Kerne an den entsprechenden mittleren Stellen ist nahezu doppelt so gross als etwas seitlich davon. Diese seitlichen, kürzeren Stäbchen werden nach der äussersten Peripherie der Netzhaut hin nur noch wenig kürzer.) Bei einem 120 Stunden dunkel gehaltenen Tiere steht das 
Pigment in der unteren Netzhautpartie vitral in dichten Knöpfehen, die durch feine Pigmentlinien mit der skleralen Pigmentlage verbunden erscheinen. Gegen die Netzhautmitte hin werden die vitralen Knöpfchen spärlicher, die feinen Pigmentlinien sind aber hier fast ebenso reichlich wie dort. In der Zone der langen Stäbchen ist das vitrale Stäbchendrittel ganz frei von Pigment, der übrige Teil enthält solches nur in geringen Mengen. Weiter oben kommt das Pignnent wieder näher an die vitralen Stäbchenenden heran, ohne sie ganz zu erreichen und ohne Knöpfchen zu bilden.

Bei einem 120 Stunden dunkel gehaltenen, dann zehn Minuten besonnten Tiere ist in der ganzen unteren (grösseren) Netzhauthälfte bis zum Streifengebiete hin das Pigment in dichten Knöpfchen innenständig, in der Nähe des Streifs werden letztere spärlicher. Die Gegend des Streifs selbst scheint an diesem Auge krankhaft verändert zu sein; sie zeigt dicke, schollige Massen mit vielen vitralen Pigmentknöpfehen. Nach oben hin steht das Pigment zunächst wieder vorwiegend skleral, weiter peripherwärts aber findet man es abermals innenständig, zum Teile sogar wiederum in dichten vitralen Knöpfehen.

Bei einem vier Stunden dunkel gehaltenen Tiere fand sich in den mittleren Netzhautpartien Pigment nur etwa im skleralen Sechstel der Stäbchen, während die inneren $5 / 6$ ganz pigmentfrei waren; die peripher sich anschliessenden Netzhautzonen zeigten Pigment in feinen Zügen in der ganzen Länge der Stäbchen; noch weiter peripher, insbesondere unten, zeigte es sich in dichten Knöpfchen auf der vitralen Netzhautoberfläche.

Bei einem $2^{1 / 2}$ Stunden der Sonne ausgesetzt gewesenen Auge löste die Hyaloidea sich überall stark pigmenthaltig ab, nur entsprechend dem Streifen war sie pigmentfrei. Das Netzhautpigment ist überall (Fig. 24), auch im ganzen Streifengebiete, in dichten Knöpfchen innenständig; im Streifen sind diese mit feinen, nach hinten gerichteten Fortsätzen versehen, dahinter ist noch eine schmale Zone dicht steheader Pigmentkörnchen zu sehen. Der Rest der Stäbchen ist bis zur Basis pigmentfrei. Ein ähnliches Verhalten fand ich bei einem Tiere, das $21 \%$ Stunden der Sonne, dann noch eine Stunde dem Tageslichte ohne direkte Sonne ausgesetzt war. Die Pigmentknöpfchen erschienen hier im Streifengebiet etwas feiner als bei dem der Sonne ausgesetzt gewesenen Tiere.

Scaeurgus (unicirrus und tetracirrus). Auch bei Scaeurgus sind die Stäbchen in den mittleren Netzhautteilen ein 
wenig länger und schmäler als oben und unten in der Peripherie, aber der Unterschied ist bei weitem nicht so gross als z. B. bei Rossia oder Eledone. Die Stäbchenlänge betrug bei einigen von mir gemessenen Exemplaren in der Mitte 0,14 mm, ein wenig seitlich davon $0,10 \mathrm{~mm}$. In den mittleren Netzhautteilen zählte ich auf $1 \mathrm{~mm}$ ca. 550 Kerne, wenig seitlich davon etwa 450 ; etwas weiter peripherwärts wurde deren Zahl wesentlich kleiner (vgl. Fig. 25).

Bei einem 24 Stunden dunkel gehaltenen Tiere war das Pigment überall gleichmässig skleralständig. Es erstreckte sich so wenig von der basalen Zone nach innen, dass fast $9 / 10$ der ganzen Stäbchenlänge pigmentfrei waren.

Bei einem $3 \frac{1}{1 / 2}$ Stunden an der Sonne gehaltenen Tiere (das aber seine Pupille ganz geschlossen gehalten hatte) war das Pigment allenthalben (vgl. Fig. 25) in dichten schwarzen Knöpfchen innenständig, auch im Gebiete des Streifs, wo die Knöpfehen viel feiner erschienen als an den Seiten. Hinter den Knöpfehen war auf einer ca. $2 \mathrm{~mm}$ breiten mittleren Netzhautzone in einem Abstande von etwa $1 / 6$ der Stäbchenlänge noch eine zweite schmale Zone mit ziemlich reichlichen feinsten Pigmentmassen sichtbar; an den seitlichen Netzhautpartien fehlten letztere. Der Unterschied im Verhalten der Limitanszellen im Streifengebiete und seitlich davon war hier besonders auffällig (vgl. Abbildung).

Todaropsis. Im anatomischen Verhalten findet sich hier insofern ein wesentlicher Unterschied von den meisten bisher beschriebenen Formen, als - ähnlich wie bei Loligo - keine mittlere Zone mit längeren und schmäleren Stäbchen vorhanden ist. Vielmehr erschienen an allen von mir untersuchten Todaropsisaugen auf vertikalen Netzhautschnitten die Stäbchen überall angenähert gleich lang und breit, vielleicht in der oberen Netzhauthälfte eine Spur länger als in der unteren. Auch die Kerne waren an allen Stellen des Schnittes bis nahe zum Rande der Netzhaut angenähert gleich zahlreich und gleichmässig verteilt. Ich fand sie auffallend viel zahlreicher als z. B. bei Rossia; an verschiedenen Stellen zählte ich tuber 1100 Kerne auf einer Strecke von $1 \mathrm{~mm}$. Sie bilden in der Regel eine mehrschichtige Zelllage von beträchtlicher Dicke, und zwar sind die äusseren Zellen mehr rund, die inneren mehr länglich, zum Teile spindelförmig, und reichen bis nahe an die Basalmembran heran. Die Limitanszellen ragen üh erall weit nach hinten aus der Pigmentzone hervor (vgl. Fig. 26). 
Bei einem moribunden Tiere, das ich noch zehn Minuten an die Sonne gebracht hatte, stand das Pigment überall fast ganz skleral; es ragte nur eine kurze Strecke zwischen die Stäbchen hinein, so dass etwa $9 / 10$ der Stäbchenlänge vollständig pigmentfrei waren.

Bei einem zwei Stunden der Sonne ausgesetzten, lebbaften Tiere fand ich das Pigment in der Peripherie der Netzhaut oben und unten in Knöpfchen innenständig, in der ganzen mittleren Netzhautpartie dagegen ungefähr in der Mitte der Stäbchen derart angehäuft, dass der mittlere Teil der Stäbchen braun erschien, während das vitrale und das sklerale Drittel fast ganz frei von Pigment waren; hier fanden sich nur vereinzelt einige Knöpfehen; die Hyaloidea war ganz pigmentfrei abgelöst und mit vielen leistenartigen Erhebungen versehen.

Bei einem drei Stunden der Sonne ausgesetzten Tiere (zeitweise war der Himmel bedeckt gewesen) fand sich (Fig. 26) in der ganzen Ausdebnung der Netzhaut das Pigment überall innenständig; ausserdem war gleichfalls fast in der ganzen Ausdehnung der Netzhaut hinter der Zone der Knöpfehen bis etwa zur Stäbchenmitte eine zweite, an verschiedenen Stellen verschieden dicht erscheinende Pigmentzone, zum Teile aus feinen, stark geschlängelten Zügen bestehend, sichtbar.

Bei einem im Dunklen gestorbenen Tiere zeigte die Netzhaut makroskopisch das in Fig. 15 wiedergegebene Bild. Mikroskopisch wurde das Pigment oben und unten ganz skleralständig gefunden, entsprechend dem braunen Streifen war es in feinen Knöpfehen auf der vitralen Netzhautfläche zu sehen. Ich halte es für wahrscheinlich, dass es sich hier um eine krankhafte Veränderung handelte.

Illex $\mathrm{Co}$ indetti. Hier liegen die Verbältnisse jenen bei Todaropsis anscheinend sehr ähnlich. Die Netzhaut erscheint auf dem Schnitte fast uberall gleichmässig dick, vielleicht wird sie in der Peripherie nach oben hin ein wenig dicker. Fine streifenförmige Verdickung der mittleren Netzhautpartien, wie bei den früher besprochenen Formen, ist nicht vorhanden. Die Kernzahl erscheint sehr gross.

Bei zwei Exemplaren, die ich moribund erhielt und noch einige Zeit an die Sonne gebracht hatte, fand ich keine Spur von phototroper Pigmentverschiebung: Das Pigment war in dichten Kölbchen an der Basis der Stäbchenreihe angehäuft und hier sehr deutlich vom stabilen Pigmente gesondert. Bei einem lebhaften Exemplar, 
das ich $1 / 2$ Stunde an die Sonne gebracht hatte, war das Pigment fast überall in feinen Knöpfchen an die vitrale Oberfläche der Stäbchen gewandert, nur in den mittleren Netzhautteilen nicht so weit wie oben und unten; es durchsetzte hier vorwiegend die vitrale Hälfte der Stäbchenreihe in feinen Zügen, doch fanden sich auch, wenngleich zienlich spärlich, vitrale Knöpfchen.

Nach den hier mitgeteilten Beobachtungen sind bisher drei verschiedene Stufen in der Ausbildung der Cephalopodennetzhaut bekannt geworden: Zunächst eine allenthalben angenähert gleichmässig dicke Netzhaut mit überall gleichmässig dicht stehenden Stäbchen (z. B. Loligo, Todaropsis). Dann Netzhäute mit einem Streifen deutlichsten Sehens, innerhalb dẹssen die Stäbchen beträchtlich länger und feiner sind als in der Umgebung (z. B. Rossia, Scaeurgus, Eledone u. a.). Endlich die von $\mathrm{Chun}$ bei Tiefseecephalopoden gefundenen Netzhäute mit einer foveaähnlichen Stelle deutlichsten Sehens, innerhalb deren die Stäbchen ausserordentlich lang und schmal gefunden werden.

Hesse war (s. o.) der Meinung, die Stäbchen seien in der Cephalopodennetzhaut überall gleichmässig verteilt. Unter dieser Voraussetzung zählte er die lichtrezipierenden Einheiten in einem bestimunten kleinen Bezirke aus und berechnete danach deren Zahl für die gesamte Netzhaut. „Wenn wir für ein Auge von Sepia vom Durchmesser des Menschenauges die Zahl der lichtrezipierenden Einheiten berechnen, so erbalten wir, die Retina als Halbkugelschale angenommen, die ausserordentliche Zahl von über 70 Millionen Stäbchen, eine Zahl, deren Bedeutung uns erst klar wird, wenn wir bedenken, dass die Zahl der rezipierenden Einheiteu für das Menschenauge inscesamt auf etwa 50 Millionen zu schätzen ist."

Nun hat sich aber gezeigt, dass bei einer Reihe von Cephalopodenarten in dem mittleren streifenförmigen Gebiete die einzelnen Elemente um etwa das Doppelte dichter stehen können als in der ganzen übrigen Netzhaut. Man,würde also einen viel zu hohen Wert erhalten, wenn die der Auszäblung zugrunde gelegte Netzhautpartie eben dem Streifengebiete angehörte, anderenfalls einen zu niedrigen. Dieser Umstand ist auch bei Beurteilung der übrigen Berechnungen über die Zahl der Stäbchen bei den anderen Cephalopodenarten mit streifenförmiger mittlerer Zone entsprechend zu berücksichtigen. Die Angabe über die Zahl der rezipierenden 
Einheiten im menschlichen Auge scheint nicht ganz zutreffend zu sein; wenigstens finde ich bei Greeff die Angabe: „Man schätzt die Anzahl der Stäbchen in der menschlichen Retina auf etwa 130 Millionen, die der Zapfen auf 7 Millionen. (W. Krause.) ${ }^{*}$

Wie gross die Abweichungen von den Tatsachen sind, die durch die vorher erwähnte Berechnungsweise herbeigeführt werden können, möge an einem Beispiele gezeigt werden: Bei einer grossen Eledone zählte ich im Gebiete des Streifs auf einer Strecke von 0,2 mm 57 Endknöpfchen, etwas seitlich davon, ausserhalb des Streifs, nur 38-40. Legen wir die erste Zahl zugrunde, so kommen im Streifengebiete auf einen Quadratmillimeter 81000 Stäbchen, ausserhalb desselben dagegen nur 40000. Bei einer Sepia zählte ich im Streifengebiete ca. 65 Endknöpfchen auf $0,2 \mathrm{~mm}$, ausserhalb nur 39 . Nach der ersten Berechnung kämen ca. 105000 Stäbchen auf den Quadratmillimeter (genau die von Hess e berechnete Zahl), ausserhalb des Streifs aber nur 40000 .

Unter den mitgeteilten Befunden scheint mir ferner von Interesse, dass bei angenähert gleichmässiger Belichtung bezw. bei Abschluss von Licht das Pigment nicht überall gleichmässig wandert, sondern in dem Gebiete des Streifs bei Belichtung langsamer vorwandert, bei Verdunklung rascher sich zurückzieht als in der übrigen Netzhaut.

Es wäre interessant, der Frage nachzugehen, inwieweit diese Pigmentverhältnisse mit der Lebensweise der Tiere zusammenhängen. Doch ist letztere bisher zu dem fraglichen Zwecke, soweit mir bekannt, noch nicht genügend genau ermittelt.

In Jatta's ${ }^{1}$ ) Monographie über Cephalopoden finde ich hinsichtlich des Tages- bezw. Nachtlebens nur für Sepia die Angabe, sie scheine eine Vorliebe für nächtliche Lebensweise zu haben, da sie gerne des Nachts auf Nahrung ansgehe.

Hesse macht die Angabe: „Die Cephalopoden mit innerer Pigmentzone sind Nachttiere und am Tage in ihrem Sehen auf den hellen Streifen der Retina beschränkt."

Wir haben gefunden, dass am hellen Lichte sämtliche untersuchte Cephalopodenarten eine innere Pigmentzone besitzen und dass bei den unter die litoralen Formen gezählten Arten sich sehr grosse Unterschiede finden, derart, dass .z. B. bei Sepia schon nach verhältnismässig geringer und kurzer Verdunklung die innere Pigmentzone fehlt, während z. B. bei Eledone selbst nach sehr langem Dunkelaufenthalte in einem grossen Teile der Netzhaut das Pigment noch in dichten Knöpfchen innenständig gefunden wird.

1) G. Jatta, I cefalopodi viventi nel golfo di Napoli. Berlin 1896. 
Hesse nimmt, wie es scheint, an, dass im Gebiete des sogen. "hellen Streifens" die Endknöpfchen überhaupt nicht von Pigment überzogen würden; denn er sagt von Sepia, Eledone und Octopus, sie sässen am Tage „untätig in ibren Schlupfwinkeln und würden in der Tat vollständig blind sein, wenn nicht im Bereich des hellen Streifens, der die Retina durchzieht, die Endknöpfchen der Neurofibrillen frei von Pigment blieben." Wir sahen aber, dass das Pigment auch im Gebiete des "hellen Streifs" die Endknöpfehen vollständig überziehen kann, ja dass bei verschiedenen der von mir untersuchten Arten bierzu nicht einmal ungewöhnlich grosse Lichtmengen nötig sind.

Dass bei solcher Innenstellung des Pigmentes die entsprechenden Netzhautpartien vollständig blind sein sollten, lässt sich wohl kaum annehmen; denn es würden dann ja bei verschiedenen Arten (z. B. Eledone u. a.), bei welchen das Pigment in den peripheren Netzhautteilen erst nach vielstündiger Verdunklung sich von den Knöpfehen zurückzieht, diese ganzen Netzhautgebiete nur selten überhaupt in Funktion treten, was bei dem sonst so zweckmässigen Baue des Sehorgans dieser Tiere wenig wahrscheinlich ist. Freilich lässt sich über die Art der Lichtwahrnehmung in jenen Fällen, wo die innere Netzhautoberfläche einen Pigmentüberzug zeigt, vorderhand noch nicht viel sagen, wenn man sich nicht in ungenügend begründeten Hypothesen verlieren will. Die Pigmenthülle, die die perzipierenden Endorgane umgibt, ist wohl sicher nicht so dicht, dass sie alles auf sie auffallende Licht vollständig absorbiert; geringe Lichtmengen werden die Stäbchenenden fast immer erreichen.

Die besondere Art der Pigmentwanderung im Streifengebiete kann nicht etwa mechanisch erklärt werden durch die Annahme, bei den dichter stehenden Stäbchen sei der Widerstand für diese Wanderung grösser; denn dann müsste ja auch das Zurückwandern bei Verdunkelung langsamer vor sich gehen. Auch die Annahme einer geringeren Reizbarkeit der Elemente im Streifengebiete scheint mir nicht hinzureichen, um diese feinen, für ein gutes Funktionieren des Sehapparates so wichtigen Vorgänge in ibren Einzelheiten genügend zu erklären.

Hesse hält es für sehr wohl möglich, dass bei Sepia, Eledone und Octopus die Endorgane im Streifengebiete weniger reizbar und deshalb für das Sehen im Dunkeln weniger tauglich seien, so dass bei nächtlichen Ausflügen der Tiere die Nachbarteile der Retina fül' 
den hellen Streifen eintreten müssten; diese Annahme ist nach unseren Befunden schon deshalb wenig wahrscheinlich, weil wir bei Verdunkelung das Pigment zuerst im Streifengebiete zurückwandern sehen und erst viel später ausserhalb des Streifens.

Auch die Frage, ob und inwieweit etwa die Verhältnisse des Sehpurpurs hier mitspielen, muss weiteren Untersuchungen überlassen bleiben. Jedenfalls ist im Cephalopodenauge der fragliche Streif nicht nachweislich purpurärmer als die ührigen Netzhautgebiete, zum Unterschiede vom menschlichen Auge, wo an der Stelle des deutlichsten Sehens das Vorkommen von Sehpurpur bisher noch nicht hat nachgewiesen werden können.

\section{Figurenerklärung.}

(Die Abbildungen sind vou Herrn Universitätszeichner W. Freytag hergestellt.)

Die Figuren 1-15 geben das makroskopische Aussehen der hinteren $A b$ schnitte von in Formol fixierten und in Alkohol nachgehärteten Cephalopodenaugen wieder.

Fig. 1. Auge einer Sepia officinalis, die 48 Stunden im Dunkeln gehalten war (siehe S. 411).

Fig. 2. Auge einer Sepia officinalis, die eine Stunde an der Sonne, danach 1/2 Stunde im Dunkeln gehalten war (siehe S. 411).

Fig. 3. Auge einer Sepia Orbignyana, die zwei Stunden dunkel gehalten war (siehe S. 413).

Fig. 4. Auge einer Sepiola Rondeletti, die $4^{1 / 2}$ Stunden dunkel gehalten war (siehe S. 413).

Fig. 5. Auge einer Eledone moscata, die im Hellen entblutet war (Fixierung in Alkohol) (siehe S. 414).

Fig. 6. Auge einer Eledone moscata, die drei Stunden an der Sonne gehalten war (siehe S. 414).

Fig. 7. Auge eines Octopus macropus, der sechs Stunden an der Sonne gehalten war (siehe S. 415).

Fig. 8. Auge eines Octopus Defilippi, der 18 Stunden dunkel gehalten war (siehe S. 415).

Fig. 9. Auge einer Loligo vulgaris, die eine Stunde dunkel, danach eine Stunde an der Sonne gehalten war (siehe S. 416).

Fig. 10. Auge einer Loligo marmorae, die im Hellen gestorben war (siehe S. 416).

Fig. 11. Auge einer Loligo vulgaris nach mässiger Belichtung (siehe S. 416).

Fig. 12. Auge einer Loligo vulgarís nach stärkerer Belichtung (siehe S. 416).

Fig. 13. Auge einer Rossia macrosoma, die 120 Stunden dunkel, danach zehn Minuten an der Sonne gehalten war (zum Teil pathologisch?) (siche S. 417). 
Fig. 14. Auge eines Scaeurgus tetracirrus, der 31/2 Stunden im Hellen gehalten war (siehe S. 417).

Fig. 15. Auge eines Todaropsis Veranyi, der nach 24 Stunden Dunkelaufenthalt tot gefunden worden war (zum Teil pathologisch?) (siehe S. 418).

Fig. 16. Schnitt durch die Netzhaut einer Sepia officinalis, die 15 Stunden dunkel gehalten war. Streifengebiet (siehe S. 425).

Fig. 17. Schnitt durch die ganze Netzhaut einer Sepia elegans, die $2 \frac{1}{2}$ Stunden dunkel gehalten war (siehe S. 426).

Fig. 18. Schnitt durch die ganze Netzhaut einer Sepia elegans, die 1/2 Stunden diffusem Lichte ausgesetzt gewesen war (siehe S. 427).

Fig. 19. Schnitt durch die seitlichen Netzhautteile einer Eledone moscata, die 20 Stunden dunkel gehalten war (siehe S. 428).

Fig. 20. Schnitt durch die Netzhaut einer Eledone moscata, die im Hellen entblutet war (Fixierung direkt in Alkohol) (siehe S. 428).

Fig. 21. Schnitt durch Streifengebiet und Nachbarschaft einer im Hellen gehaltenen Eledone (siehe S. 428).

Fig. 22. Schnitt durch die ganze Netzhaut eines Octopus Defilippi, der erst 48 Stunden dunkel, dann eine Viertelstunde in diffusem Lichte gehalten war (siehe S. 429).

Fig. 23. Flächenpräparat der spontan abgelösten Membrana hyaloidea eines im Hellen gehaltenen Octopus (siehe S. 429).

Fig. 24. Schnitt durch die mittleren Netzhautteile (Streifen) einer Rossia macrosoma, die zwei Stunden der Sonne ausgesetzt gewesen war (siehe S. 431).

Fig. 25. Schnitt durch die mittleren (rechts) und seitlichen (links) Netzhautteile eines Scaeurgus, der $31 / 2$ Stunden dem Sonnenlichte ausgesetzt gewesen war (siehe S. 439).

Fig. 26. Schnitt durch die Netzhaut eines Todaropsis Veranyi, der drei Stunden der Sonne ausgesetzt gewesen war (siehe S. 433). 
Fig.1.
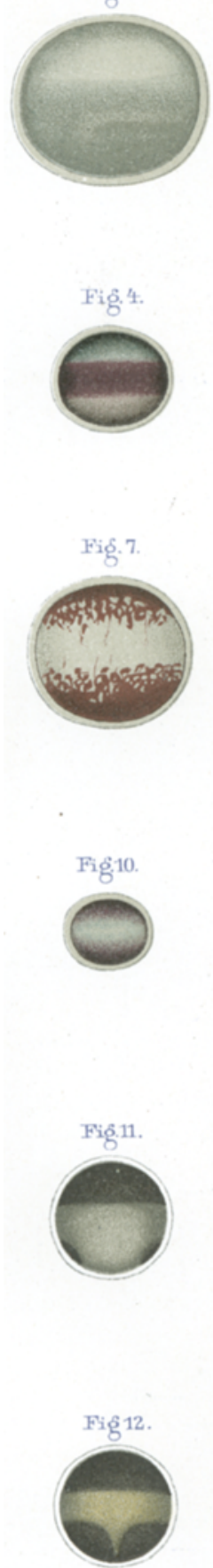
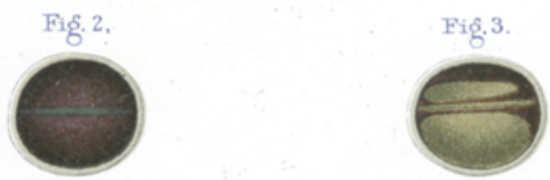

Fig. 5.

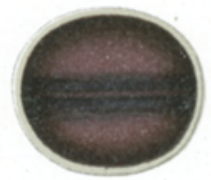

Fig. 6.

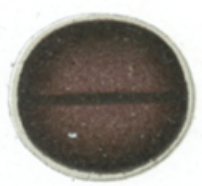

Fig. 9.

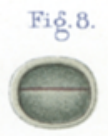

Fig. 16.
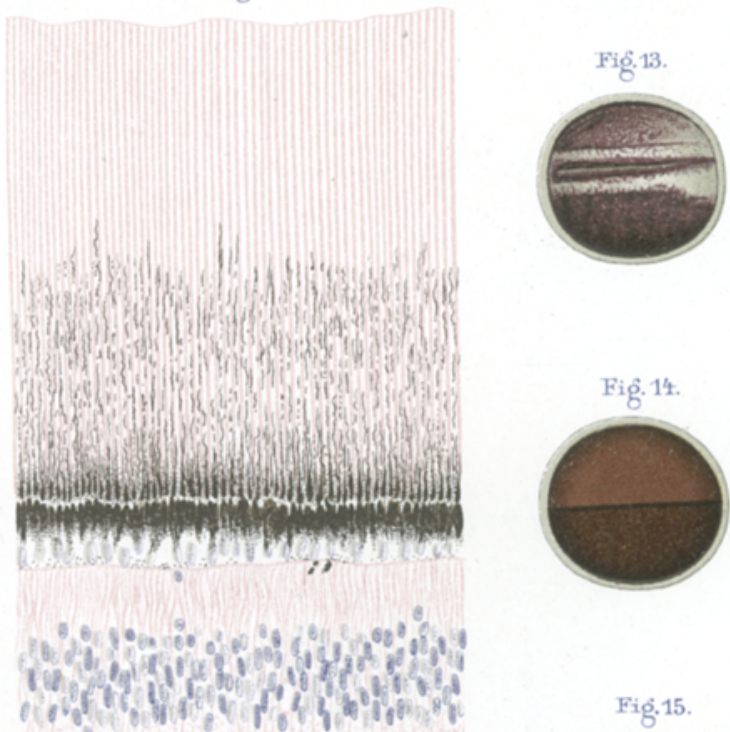

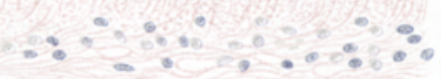

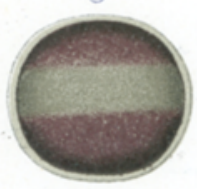$$
\text { . }
$$ 


\section{Fig. 17.}

$\infty$

7. Fed
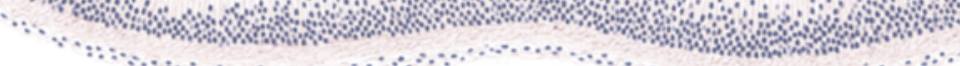

Fig.18.

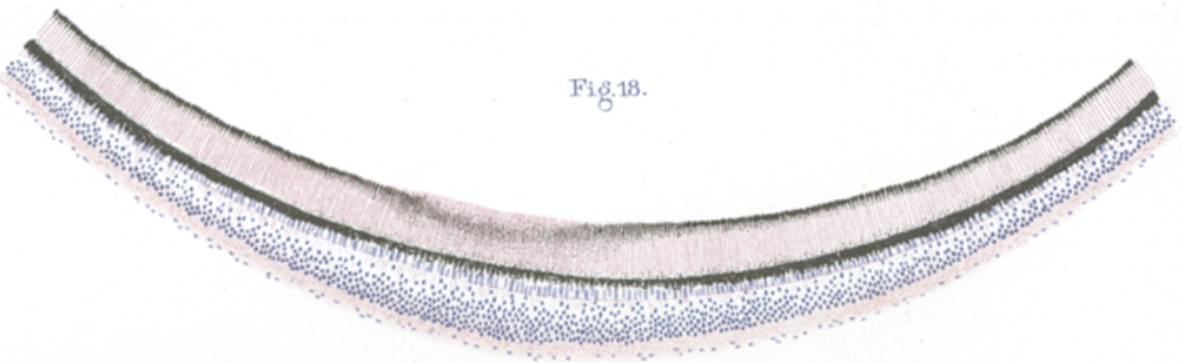

Fig.19.

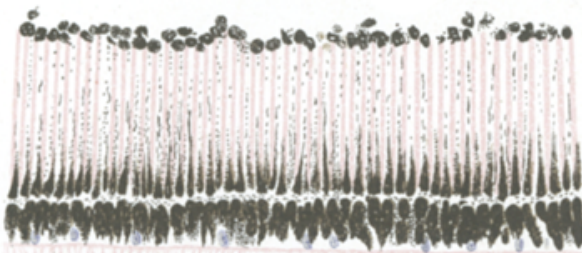

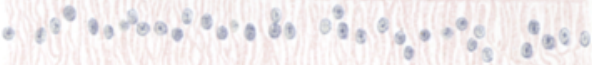

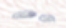

Fig.20.

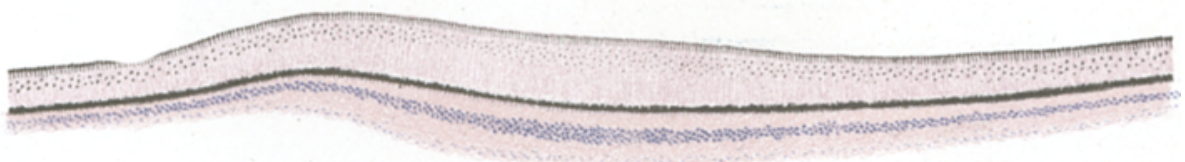

W. Freytag grez. 

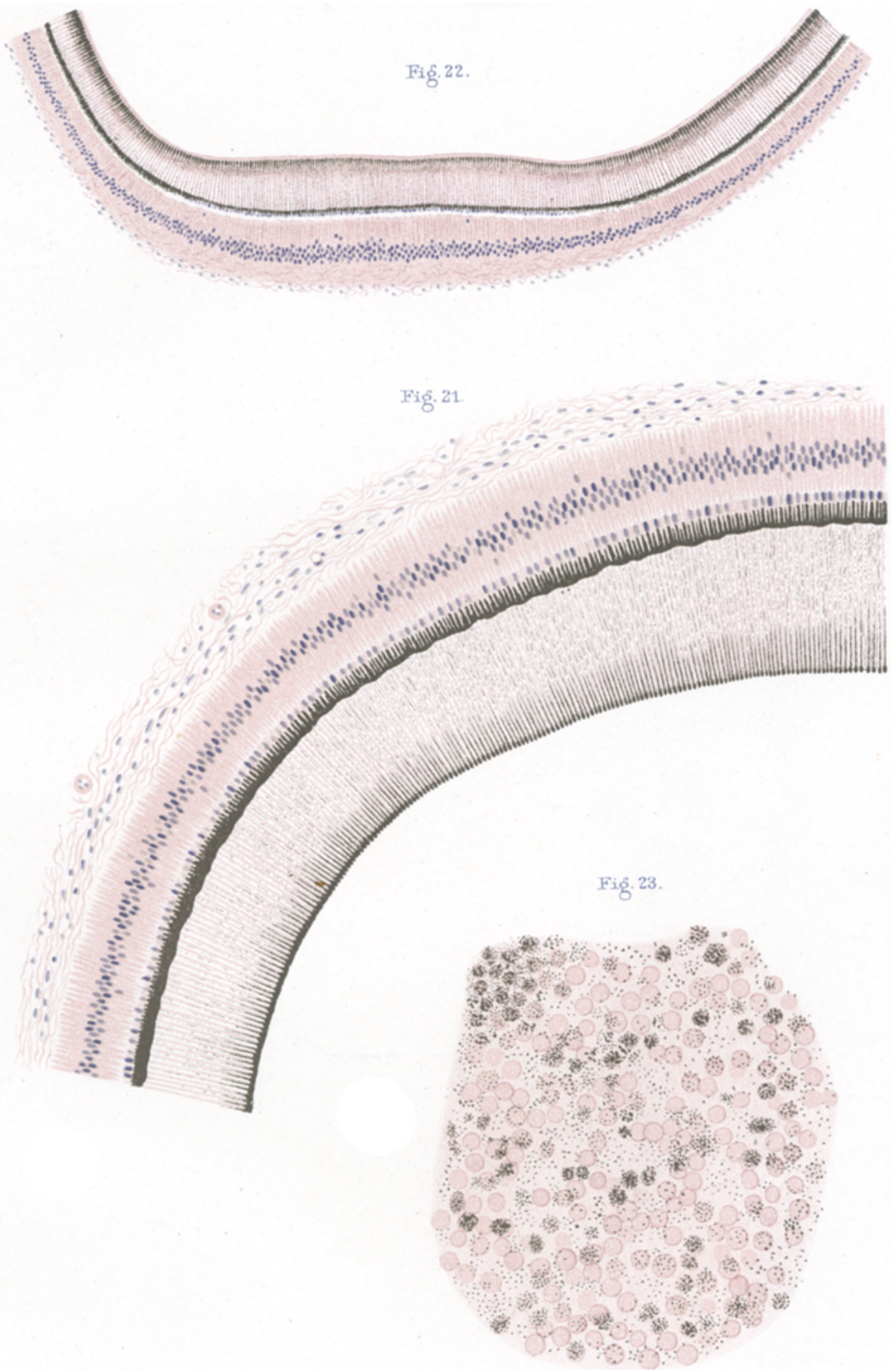
Fig. 24 .

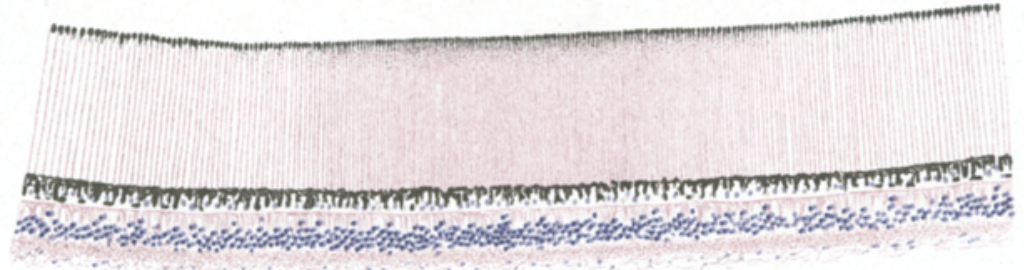

Fig. 25.
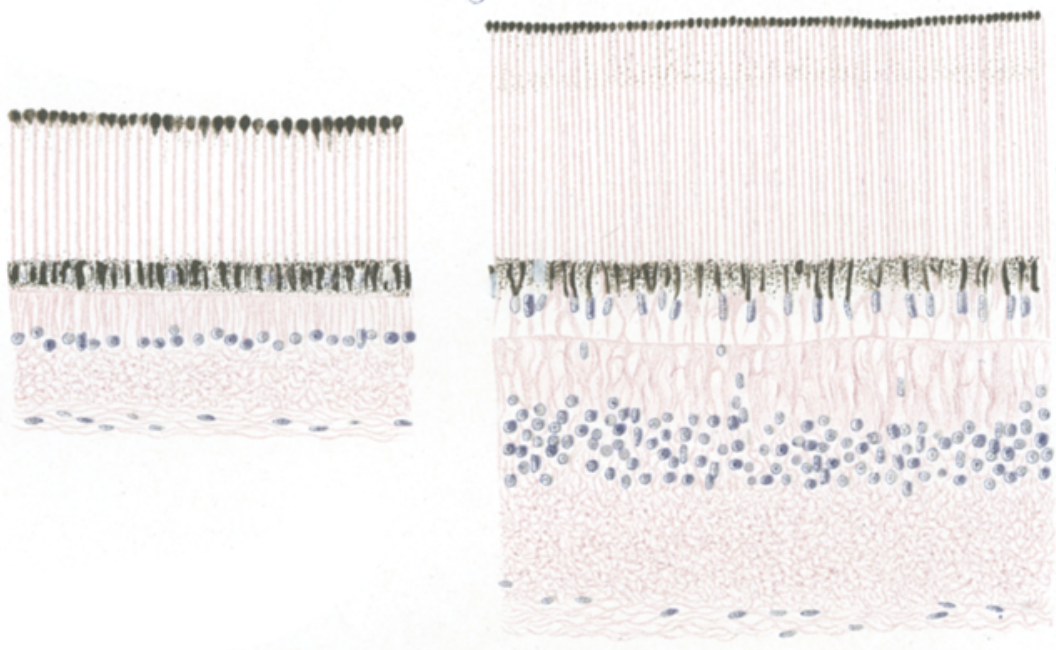

Fig. 26.

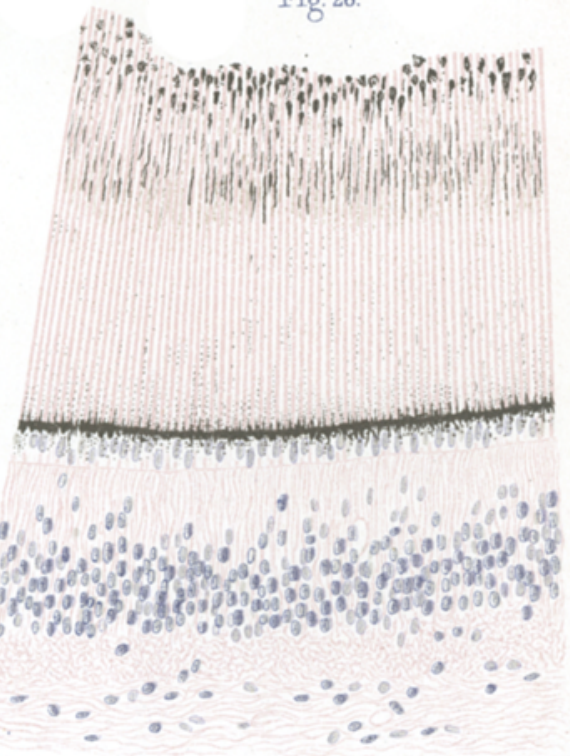

